\title{
On the Interplay Between Geometrical and Analytical Properties of Functions of Bounded Boundary Rotation*
}

\section{WOLFRAM KOEPF}

Freie Universität Berlin, Fachbereich Mathematik, Ârnimallee 3, 1000 Berlin 33, Germany

We shall give geomeirical interpretations of analytical terms depending on the logarithmic derivative $f^{\prime \prime} / f^{\prime}$ and the Schwarzian derivative $\left(f^{\prime \prime} / f^{\prime}\right)^{\prime}-\frac{1}{2}\left(f^{\prime \prime} / f^{\prime}\right)^{2}$ for certain locally univalent functions $f$ in the unit disk, especially for functions of bounded boundary rotation.

AMS No. 30C45, 30C80, 30C50, 30C75

Communicated: R. P. Gilbert

(Received September 11, 1990)

\section{UNIVALENT FUNCTIONS}

We consider functions that are analytic in the unit disk

$$
\mathrm{D}:=\{z \in \mathbb{C}|| z \mid<1\} \text {. }
$$

A function is called univalent (or schlicht) if it is one-to-one. The Riemann mapping theorem guarantees the existence of a univalent map $f: D \rightarrow G$ for each simply connected plain domain $G \neq C$. Moreover $f$ with given $f(0)$ is uniquely determined except of the composition with rotations $z \mapsto e^{i \alpha} z$ of $\mathrm{D}$.

If we speak about convergence of a sequence $\left(f_{n}\right)$ of analytic functions, we mean locally uniform convergence and write $f_{n} \rightarrow f$. The family $A$ of analytic functions of $\mathrm{D}$ together with this topology is a Fréchet space, i.e. a locally convex complete metrizable linear space.

A sequence of univalent functions not converging locally uniformly to $\infty$ is normal, and there is a convergent subsequence. The limit function is univalent or constant.

The family $S$ of univalent functions that are normalized by $f(0)=0, f^{\prime}(0)=1$, i.e.

$$
f(z)=z+a_{2} z^{2}+a_{3} z^{3}+\cdots,
$$

is a compact subset of $A$.

A function $f \in A$ is called $m$-fold symmetric if it has the special form $(m \in \mathbf{N})$

$$
f(z)=z+a_{m+1} z^{m+1}+a_{2 m+1} z^{2 m+1}+\cdots
$$

*This work is part of the author's "Habilitationsschrift" accepted by the Free University of Berlin in July 1990. 
which is equivalent to the fact that the Riemann image surface $F$ is $m$-fold symmetric with respect to the origin, i.e. for all $w \in F, k=1, \ldots, m$ also the points $e^{2 \pi i k / m} w \in F$. (References: [6], [10], [32].)

\section{FUNCTIONS WITH POSITIVE REAL PART}

Let $P$ denote the subset of $A$ of functions $p$ with positive real part that are normalized by $p(0)=1$.

A function of the form

$$
p(z)=\int_{\partial \mathbf{D}} \frac{1+x z}{1-x z} d \mu(x),
$$

where $\mu$ denotes a Borel probahility measure on $\partial \mathbf{D}$, clearly has positive real part, because the kernel functions have this property. The famous Herglotz representation theorem states that the converse is also true. This is equivalent to the fact that the extreme points of $P$ (i.e. the points which have no proper convex representation within the convex set $P$ ) are the kernel functions of representation (3), which map D univalently onto the right half-plane $\{w \in \mathbb{C} \mid \operatorname{Re} w>0\}$ (see e.g. [33], [12]); we write $E(P)=\{(1+x z) /(1-x z) \mid x \in \partial 0\}$. By the Krein-Milman theorem their closed convex hull $\overline{\mathrm{co}}(\mathrm{E} P)$ is all of $P$ and so their convex hull $\operatorname{co}(\mathrm{E} P)$ lies dense in $P$ with respect to the topology of locally uniform convergence (which makes $P$ compact), so that each function $p \in P$ can be locally uniformly approximated by functions $p_{n}$ of the form

$$
\begin{aligned}
& p_{n}(z)=\sum_{k=1}^{n} \mu_{k} \frac{1+x_{k} z}{1-x_{k} z}, \quad\left|x_{k}\right|=1, \quad \mu_{k}>0 \quad(k=1, \ldots, n), \\
& \sum_{k=1}^{n} \mu_{k}=1, \quad n \in \mathrm{N} .
\end{aligned}
$$

The functions of the form (4) give the so-called Carathéodory boundary of $P$.

A function $f$ is called subordinate to $g$, if $f=g \circ \omega$ for some function $\omega \in A$ with $\omega(0)=0$ and $\omega(\mathrm{D}) \subset \mathbf{D}$; we write $f \prec g$. The subordination principle states that if $g$ is univalent then $f \prec g$ if and only if $f(0)=g(0)$ and $f(D) \subset g(D)$, and so $p \in P$ iff $p \prec(1+z) /(1-z)$. If $f \prec g$ then by Schwarz's Lemma $f\left(\mathbf{D}_{r}\right) \subset g\left(\mathbf{D}_{r}\right)$ for all $r \in] 0,1\left[\right.$ where $\mathrm{D}_{r}:=\{z \in \mathbb{C}|| z \mid<r\}$.

By $B$ we denote the family of functions $\omega \in A$ with $\omega(0)=0$ and $\omega(\mathbf{D}) \subset \mathbf{D}$.

A compact family which is similar to $P$ is the class $\tilde{P}$ of functions $p$ normalized by $p(0)=1$ for which there is some $\alpha \in \mathbf{R}$ such that the real part of $e^{i \alpha} p$ is positive. One sees that $p \in \tilde{P}$ iff $p \prec(1+y z) /(1-z)$, where $y=e^{-2 i \alpha}$.

\section{POLYGONS AND SCHWARZ-CHRISTOFFEL MAPPINGS}

Let $f \in A$ be continuous in $\bar{D}$ and have a Riemann surface $F$ as image domain whose boundary consists of a finite number of linear arcs, such that the boundary correspondence $\partial \mathrm{D} \rightarrow \partial F$ is one-to-one. Then $F$ is called a polygon. Let $F$ have $n$ 
vertices of inner angles $\alpha_{k} \pi(k=1, \ldots, n)$. We do not suppose $f$ to be univalent, so that $\alpha_{k}>2$ is possible, whereas for univalent polygons

$$
\alpha_{k} \leq 2 \quad(k=1, \ldots, n)
$$

If we have a bounded vertex then

$$
\alpha_{k}>0
$$

If a vertex lies at infinity we measure the angle on the Riemann sphere and have

$$
\alpha_{k} \geq 0
$$

where $\alpha_{k}=0$ is a zero angle which corresponds to two paraliel rays of $\partial F$.

Let now $x_{k}$ be the prevertices, i.e. the preimages under $f$ of the vertices $f\left(x_{k}\right)$. Then the Schwarz-Christoffel formula is the representation

$$
\frac{f^{\prime \prime}}{f^{\prime}}(z)=-2 \sum_{k=1}^{n} \frac{\mu_{k}}{z-x_{k}}
$$

where

$$
2 \mu_{k} \pi:= \begin{cases}\left(1-\alpha_{k}\right) \pi & \text { if } f\left(x_{k}\right) \text { is bounded } \\ \left(1+\alpha_{k}\right) \pi & \text { if } f\left(x_{k}\right) \text { is unbounded }\end{cases}
$$

denote the outer angles. The formula

$$
\sum_{k=1}^{n} \mu_{k}=1
$$

corresponds in the bounded (univalent) case both to the rule for the sum of angles in an $n$-gon and to the fact that the increment of the tangent direction is exactly $2 \pi$ when surrounding the polygon on $\partial F$ one time.

On the other hand, if $f$ fulfills (8) and (10) with $x_{k} \in \partial \mathrm{D}$ for $k=1, \ldots, n$, then the Riemann image surface $f(\mathrm{D})$ is a polygon.

If $f\left(x_{k}\right)$ is bounded then relation (6) yields

$$
\mu_{k}<\frac{1}{2}
$$

whereas for unbounded $f\left(x_{k}\right)$ relations (7) and (9) give

$$
\mu_{k} \geq \frac{1}{2}
$$

If $f$ is univalent, then (5) leads to

$$
\mu_{k} \geq-\frac{1}{2} \quad(k=1, \ldots, n) .
$$

(References: [20], [35].) 


\section{CONVEX FUNCTIONS}

A function $f \in A$ is called convex if it maps $\mathrm{D}$ univalently onto a convex domain. Therefore it is necessary and sufficient that

$$
1+z \frac{f^{\prime \prime}}{f^{\prime}} \in P
$$

Let $K$ denote the family of convex functions that are normalized by (1).

\section{FUNCTIONS OF BOUNDED BOUNDARY ROTATION}

The boundary rotation of a polygon $F$ is the total change of the tangent direction when surrounding the boundary of the polygon one time and can be calculated as the sum of the absolute value of the outer angles

$$
\operatorname{br}(F)=\sum_{k=1}^{n} 2\left|\mu_{k}\right| \pi .
$$

The boundary rotation of the corresponding Schwarz-Christoffel mapping is defined to be the boundary rotation of its image polygon. A function $f$ has boundary rotation $K \pi$, if it can be approximated by Schwarz-Christoffel mappings with respect to locally uniform convergence, i.e. if

$$
\frac{f^{\prime \prime}}{f^{\prime}}(z)=-2 \int_{\partial \mathbf{D}} \frac{d \mu(x)}{z-x}
$$

where $\mu$ is a signed measure on $\partial D$ with the properties

$$
\int_{O \mathbf{D}} d \mu(x)=1
$$

and

$$
\operatorname{br}(f)=2 \pi \int_{\partial \mathbf{D}}|d \mu(x)|=K \pi
$$

Representation (16) is called the Paatero representation of $f$.

Let $V(K)$ denote the family of functions of bounded boundary rotation at most $K \pi$ that are normalized by (1). So $V(K)$ is the locally uniform closure of the corresponding family of normalized Schwarz-Christoffel mappings of bounded boundary rotation at most $K \pi$.

Generalized polygons with an infinite number of vertices $w_{k}(k \in \mathbf{N})$ of outer angle $2 \mu_{k} \pi$ with $\sum_{k=1}^{\infty}\left|\mu_{k}\right|<\infty$ are examples of functions of bounded boundary rotation. (References: [27], [35], [13].) 


\section{LINEARLY ACCESSIBLE DOMAINS AND CLOSE-TO-CONVEX FUNCTIONS}

A domain $F$ is called (angularly) accessible of order $\beta(\beta \in[0,1])$, if it is the complement of the union of rays that are pairwise disjoint except that the origin of one ray may lie on another one of the rays, such that every ray is the bisector of a sector of angle $(1-\beta) \pi$ which wholly lies in the complement of $F$. If $\beta=1$ then $F$ is called (strictly) linearly accessible (see [4], [34], [31]). A function $f$ is called close-10-convex of order $\beta(\beta \geq 0)$, if there exist a convex function $g$ and a function $p \in \tilde{P}$ such that the representation

$$
f^{\prime}=g^{\prime} \cdot p^{\beta}
$$

holds. It turns out that for $\beta \in[0,1] f$ is close-to-convex of order $\beta$ if and only if $f$ is univalent and $f(D)$ is accessible of order $\beta$ (see e.g. [19]).

\section{INVARIANTS UNDER SIMILARITIES AND THE NEHARI CRITERION}

If $f \in S$, i.e. $f$ is a univalent function that is normalized by (1), then the renor malized composition $g$ of $f$ with a univalent automorphism $\omega: \mathrm{D} \rightarrow \mathrm{D}$ of the unit disk

$$
\omega(z):=x \frac{z+a}{1+\bar{a} z}, \quad a \in \mathrm{D}, \quad|x|=1,
$$

given by

$$
g:=\frac{f \circ \omega-f \circ \omega(0)}{(f \circ \omega)^{\prime}(0)},
$$

lies in S. Pommerenke [29]-[30] called families with this property linearly invariant, and showed that many results about univalent functions are effected by this property. The function $g$ is called Koebe transform of $f$, it has (in the univalent case) a range $G$ which is similar to the range $F$ of $f$, i.e. $G=a F+b(a, b \in \mathbb{C})$, and all normalized functions with a similar domain have this form. The second coefficient of $g$ has for $x=1$ absolute value

$$
\kappa(f ; a):=\left|a_{2}(g)\right|=\left|-\bar{a}+\frac{1}{2}\left(1-|a|^{2}\right) \frac{f^{\prime \prime}}{f^{\prime}}(a)\right| .
$$

We call $\kappa$ the Koebe expression of $f$.

For a locally univalent function $f$ we define the order of $f$ by

$$
\operatorname{ord}(f):=\sup _{a \in \mathbf{D}} \kappa(f ; a) .
$$

It represents the order of the linearly invariant family $\operatorname{Lin}(f)$ generated by $f$, and it is bounded if and only if $\operatorname{Lin}(f)$ is normal (see [29], Folgerung 1.1).

For an analytical expression to have a geometrical meaning the expression must have a certain invariance property with respect to the composition with automorphisms of $\mathrm{D}$, because the range is invariant under this composition. 
We have for the Koebe expression

LEMMA 1 If $f \in A$ is locally univalent and $\omega$ is defined by (20), then for $g=f \circ \omega$ holds

$$
\kappa(g ; z)=\kappa(f ; \omega(z)) \quad(z \in \mathbf{D})
$$

Proof The relations

$$
\frac{g^{\prime \prime}}{g^{\prime}}=\omega^{\prime} \frac{f^{\prime \prime}}{f^{\prime}}+\frac{\omega^{\prime \prime}}{\omega^{\prime}}
$$

and

$$
1-|\omega(z)|^{2}=1-\frac{z+a}{1+\bar{a} z} \frac{\bar{z}+\bar{a}}{1+a \bar{z}}=\frac{\left(1-|z|^{2}\right)\left(1-|a|^{2}\right)}{(1+\bar{a} z)(1+a \bar{z})}
$$

imply

$$
\begin{aligned}
& r(g ; z)^{2}=\left|\bar{z}+\frac{1}{2}\left(1 \quad|z|^{2}\right)\left(x \frac{1-|a|^{2}}{(1+\bar{a} z)^{2}} \frac{f^{\prime \prime}}{f^{\prime}}(\omega)-\frac{2 \bar{a}}{1+\bar{a} z}\right)\right|^{2} \\
& =\left(\begin{array}{c}
\bar{z}+\bar{a} \\
1+\bar{a} z
\end{array}+\frac{1\left(1-|z|^{2}\right)\left(1-|a|^{2}\right)}{2} x^{f^{\prime \prime}}(1+\bar{a} z)^{2}(\omega)\right) \\
& \cdot\left(-\frac{z+a}{1+a \bar{z}}+\frac{1}{2} \frac{\left(1-|z|^{2}\right)\left(1-|a|^{2}\right)}{(1+a \bar{z})^{2}} \bar{x}\left(\frac{f^{\prime \prime}}{f^{\prime}}(\omega)\right)\right) \\
& =|\omega|^{2}-\frac{1}{2} \frac{\left(1-|z|^{2}\right)\left(1-|a|^{2}\right)}{(1+\bar{a} z)(1+a \bar{z})}\left(\frac{\bar{z}+\bar{a}}{1+a \bar{z}} \overline{\left(\frac{f^{\prime \prime}}{f^{\prime}}(\omega)\right)}+\frac{z+a}{1+\bar{a} z} x \frac{f^{\prime \prime}}{f^{\prime}}(\omega)\right) \\
& +\frac{1}{4} \frac{\left(1-|z|^{2}\right)^{2}\left(1-|a|^{2}\right)^{2}}{(1+\bar{a} z)^{2}(1+a \bar{z})^{2}}\left|\frac{f^{\prime \prime}}{f^{\prime}}(\omega)\right|^{2}=\kappa(f ; \omega(z))^{2} \text {. }
\end{aligned}
$$

Moreover, $\kappa$ as a function of $\int$ does only depend on $f^{\prime \prime} / f^{\prime}$, so that it is also invariant under similarities of the range. From this it followsp 1 (for univalent $f$ ) the expressions

$$
\operatorname{ord}(f) \quad \text { and } \quad \inf _{a \in \mathbf{D}} \kappa(f ; a)
$$

as well as

$$
\limsup _{a \rightarrow O \mathbf{D}} \kappa(f ; a) \quad \text { and } \quad \liminf _{a \rightarrow \partial \mathbf{D}} \kappa(f ; a)
$$

represent geometric properties which are invariant under similarities.

The same is true for the expression $\sigma$, which is defined with the aid of the Schwarzian derivative $S_{f}$ of $f$, i.e.

$$
S_{f}:=\left(\frac{f^{\prime \prime}}{f^{\prime}}\right)^{\prime}-\frac{1}{2}\left(\frac{f^{\prime \prime}}{f^{\prime}}\right)^{2}
$$

namely $(a \in \mathbf{D})$

$$
\sigma(f ; a):=\left(1-|a|^{2}\right)^{2}\left|S_{f}(a)\right|
$$


LEMMA 2 If $f \in A$ is locally univalent and $\omega$ is defined by (20), then for $g=f$ ow holds

$$
\sigma(g ; z)=\sigma(f ; \omega(z)) \quad(z \in \mathbf{D}) .
$$

Proof The well-known invariance property of the Schwarzian derivative

$$
S_{g}=S_{f}(\omega) \cdot\left(\omega^{\prime}\right)^{2}
$$

implies the result similarly as in the above case.

We call $\sigma$ the Nehari expression of $f$, because Nehari has shown that $\sigma(f ; z) \leq 2$ implies univalence, and on the other hand univalent functions satisfy $\sigma(f ; z) \leq 6$. Moreover, convex functions fuifill $\sigma(f ; z) \leq 2$ (see [25], [26] and [21]).

\section{LOGARITHMIC DERIVATIVE AND THE BECKER CRITERION}

Another important univalence criterion involves the logarithmic derivative and is due to Becker. We call $(a \in \mathrm{D})$

$$
\lambda(f ; a):=\left(1-|a|^{2}\right)\left|\frac{f^{\prime \prime}}{f^{\prime}}(a)\right|
$$

the Becker expression of $f$. Beckers criterion states that $\lambda(f ; z) \leq 1$ implies the univalence of $f$. On the other hand univalent functions satisfy $\lambda(f ; z) \leq 6$ (see [2]).

Let us note the following correspondence between the Nehari and Becker conditions.

LEMMA 3 Let $f \in A$ be locally univalent. Then

$$
\begin{aligned}
& \sup _{z \in \mathbf{D}} \lambda(f ; z) \leq \lambda \Rightarrow \sup _{z \in \mathbf{D}} \sigma(f ; z) \leq 4 \lambda+\frac{1}{2} \lambda^{2}, \\
& \sup _{z \in \mathbf{D}} \sigma(f ; z) \leq \sigma \Rightarrow \sup _{z \in \mathbf{D}} \lambda(f ; z) \leq 2\left(1+\frac{\sigma}{2}\right)^{1 / 2}+2 .
\end{aligned}
$$

Proof Statement (a) is proved in [7]. (A sharper version of it is given in [37]). For to prove (b) observe that the functions $f$ satisfying $\sigma(f ; z) \leq \sigma(z \in \mathrm{D})$ form a linearly invariant family of order $(1+\sigma / 2)^{1 / 2}$ (see [29], Folgerung 2.3). Therefore $\kappa(f ; z) \leq(1+\sigma / 2)^{1 / 2}$ (see [29], Lemma 1.2) which implies the result.

\section{THE KOEBE, NEHARI AND BECKER EXPRESSIONS FOR POLYGONS}

Let $F=f(\mathrm{D})$ be a polygon with inner angles $\alpha_{k} \pi(k=1, \ldots, n)$, so that $f$ has a Schwarz-Christoffel representation

$$
\begin{aligned}
& \frac{f^{\prime \prime}}{f^{\prime}}(z)=-2 \sum_{k=1}^{n} \frac{\mu_{k}}{z-x_{k}}, \quad\left|x_{k}\right|=1 \quad(k=1, \ldots, n), \\
& \sum_{k=1}^{n} \mu_{k}=1
\end{aligned}
$$


where $2 \mu_{k} \pi(k=1, \ldots, n)$ are the outer angles $(9)$ and $x_{k}(k=1, \ldots, n)$ are the prevertices.

We write $z=r e^{i \theta}$ and define

$$
b_{k}(z):=\frac{1-\bar{z} x_{k}}{z-x_{k}}
$$

Obviously $\left|b_{k}\right|=1(k=1, \ldots, n)$ for all $z \in \mathbf{D}$. We get then for the Koebe, Becker and Nehari expressions

$$
\begin{aligned}
\kappa(f ; z) & =\left|-\bar{z}+\frac{1}{2}\left(1-|z|^{2}\right) \frac{f^{\prime \prime}}{f^{\prime}}(z)\right| \\
& =\left|\bar{z}+(1+r) \sum_{k=1}^{n} \frac{1-r}{z-x_{k}} \mu_{k}\right|=\left|\sum_{k=1}^{n} \mu_{k} h_{k}(z)\right|, \\
\lambda(f ; z) & =\left(1-|z|^{2}\right)\left|\frac{f^{\prime \prime}}{f^{\prime}}(z)\right|=2(1+r)\left|\sum_{k=1}^{n} \frac{1-r}{z-x_{k}} \mu_{k}\right|,
\end{aligned}
$$

and, since for the Schwarzian derivative one has

$$
\begin{aligned}
S_{f}(z) & =2 \sum_{k=1}^{n} \frac{\mu_{k}}{\left(z-x_{k}\right)^{2}}-2\left(\sum_{k=1}^{n} \frac{\mu_{k}}{z-x_{k}}\right)^{2} \\
& =\sum_{j, k=1}^{n} \mu_{j} \mu_{k}\left(\frac{1}{z-x_{j}}-\frac{1}{z-x_{k}}\right)^{2}
\end{aligned}
$$

finally

$$
\begin{aligned}
\sigma(f ; z) & =\left(1-|z|^{2}\right)^{2}\left|S_{f}(z)\right| \\
& =2(1+r)^{2}\left|\sum_{k=1}^{n}\left(\frac{1-r}{z-x_{k}}\right)^{2} \mu_{k}-\left(\sum_{k=1}^{n} \frac{1-r}{z-x_{k}} \mu_{k}\right)^{2}\right| \\
& =\left|\sum_{j, k=1}^{n} \mu_{j} \mu_{k}\left(b_{j}(z)-b_{k}(z)\right)^{2}\right|
\end{aligned}
$$

The following lemma will be used to examine the boundary behaviour of these expressions.

LEMMA 4 Let $\left|x_{k}\right|=1$, then

$$
\lim _{r \rightarrow 1} \frac{1-r}{r e^{i \theta}-x_{k}}=\left\{\begin{array}{cl}
-\overline{x_{k}} & \text { if } \theta=\arg x_{k} \\
0 & \text { otherwise }
\end{array} .\right.
$$

Proof We have

$$
\frac{1-r}{r e^{i \theta}-x_{k}}=-\overline{x_{k}}(1-r) \frac{1}{1-r e^{i \theta} \overline{x_{k}}}
$$


If now $e^{i \theta} \neq x_{k}$, then the last fraction is bounded, so that the right hand side tends to zero, while for $e^{i \theta}=x_{k}$ we have $(1-r) /\left(1-r e^{i \theta} \overline{x_{k}}\right)=1$.

Therefore we get from $(30)-(33)$ :

LEMMA 5 If $f$ is a Schwar-Christoffel mapping (28), then

$$
\begin{aligned}
& \lim _{r \rightarrow 1} \kappa\left(f ; r e^{i \theta}\right)=\left\{\begin{array}{cc}
\left|1-2 \mu_{k}\right|=\alpha_{k} & \text { if } \theta=\arg x_{k} \\
1 & \text { otherwise }
\end{array},\right. \\
& \lim _{r \rightarrow 1} \lambda\left(f ; r e^{i \theta}\right)=\left\{\begin{array}{cc}
4\left|\mu_{k}\right| & \text { if } \theta=\arg x_{k} \\
0 & \text { otherwise },
\end{array}\right. \\
& \lim _{r \rightarrow 1} \sigma\left(f ; r e^{i \theta}\right)=\left\{\begin{array}{cc}
8\left|\mu_{k}\left(1-\mu_{k}\right)\right|=2\left|1-\alpha_{k}^{2}\right| & \text { if } \theta=\arg x_{k} \\
0 & \text { otherwise }
\end{array}\right.
\end{aligned}
$$

Now it follows

THEOREM 1 If $f$ is a Schwarz-Christoffel mapping (28), then

$$
\begin{aligned}
& \limsup _{z \rightarrow O \mathbf{D}} \kappa(f ; z)=\max _{0 \leq k \leq n}\left|1-2 \mu_{k}\right|=\max _{0 \leq k \leq n} \alpha_{k}, \\
& \liminf _{z \rightarrow O \mathbf{D}} \kappa(f ; z)=\min _{0 \leq k \leq n}\left|1-2 \mu_{k}\right|=\min _{0 \leq k \leq n} \alpha_{k}, \\
& \limsup _{z \rightarrow O \mathbf{D}} \lambda(f ; z)=4 \max _{1 \leq k \leq n}\left|\mu_{k}\right|, \\
& \limsup _{z \rightarrow \theta \mathbf{D}} \sigma(f ; z)=8 \max _{1 \leq k \leq n}\left|\mu_{k}\left(1-\mu_{k}\right)\right|=2 \max _{1 \leq k \leq n}\left|1-\alpha_{k}^{2}\right|,
\end{aligned}
$$

where $\mu_{0}:=0$ and $\alpha_{0}:=1$.

We remark that (a) can be interpreted in the following way: the limsup of the Koebe expression measures the largest inner angle divided by $\pi$, where we have to take into consideration the angle $\pi$ of each smooth boundary point, whereas the liminf of the Koebe expression measures the smallest inner angle divided by $\pi$. It is a special property of polygons that every boundary point is either smooth or a vertex. We shall see later that these considerations can be generalized to a larger class of functions whose images have this property, namely to functions with bounded boundary rotation.

On the other hand, by reason of (11)-(12) the limsup of the Becker expression measures whether the polygon is bounded:

COROLlARY 1 If $f$ is a Schwarz-Christoffel mapping (28), and if $\alpha_{k} \leq 2(k=$ $1, \ldots, n$ ) (in particular, if $f$ is univalent), then

$$
\limsup _{z \rightarrow O 0} \lambda(f ; z) \geq 2 \Leftrightarrow f \text { is unbounded. }
$$




\section{DOMAINS WITH THE ANGLE PROPERTY}

Let $F$ be a simply connected plain domain or Riemann surface. Then we say that $F$ has the angle property, if each boundary point is either smooth, i.e. there is a tangent there, or it is a vertex, i.e. there exist two halftangents corresponding to the left and right derivatives of some parametric representation of the boundary curve. An analytic function $f: D \rightarrow F$ which extends continuously to the boundary of $D$ has the angle property if its Riemann image surface $F$ has it.

If $F$ has the angle property, then at each boundary point we define the inner angle to be the angle between the halftangents measured from the interior of $F$. The inner angle always exists and equals $\pi$ at each smooth boundary point. With $\alpha_{\max } \pi$ and $\alpha_{\min } \pi$ we denote the supremum and the infimum of the inner angles of $F$ and we speak about the largest and the smallest inner angle of $F$.

The definitions also apply if $F$ is unbounded considering tangents and halftangents on the Rienanin spherc. An unbounded $F$ with the argle properity indust have an inner angle also at each point on $\partial F$ which is unbounded.

The outer angle at some vertex is defined as in the case of polygons by (9), and its absolute value measures the change of the tangent direction at the vertex discarding the direction of the change. The outer angle at some smooth boundary point equals zero. By $2 \mu_{\max } \pi$ and $2 \mu_{\min } \pi$ we denote the supremum and the infimum of the absolute value of the outer angles of $F$. Remark that in the unbounded case the outer angle has not the same geometrical meaning as in the bounded case, in particular if $\infty$ is a smooth boundary point, then the corresponding outer angle $2 \mu_{k} \pi$ does not equal zero but equals $2 \pi$.

\section{THE KOEBE, NEHARI AND BECKER EXPRESSIONS FOR FUNCTIONS OF BOUNDED BOUNDARY ROTATION}

In this section we generalize some of the results for Schwarz-Christoffel mappings to functions of bounded boundary rotation. It is a result essentially due to Paatero that functions of bounded boundary rotation have the angle property (see [27]), so that there exist the largest and the smallest inner and outer angles $\alpha_{\max } \pi, \alpha_{\min } \pi$, $2 \mu_{\max } \pi$ and $2 \mu_{\min } \pi$. This result is contained in the following

THEOREM 2 Let $f \in V(K)$ have boundary rotation $K \pi$. Then $f$ has the Paatero representation

$$
\frac{f^{\prime \prime}}{f^{\prime}}(z)=-2 \int_{\partial \mathbf{D}} \frac{d \mu(x)}{z-x}, \quad \int_{\partial \mathbf{D}} d \mu(x)=1, \quad \int_{\partial \mathbf{D}}|d \mu(x)|=\frac{K}{2}
$$

for some signed measure $\mu$ on $\partial \mathrm{D}$, and it has a spherically continuous extension $f$ : $\overline{\mathrm{D}} \rightarrow \hat{\mathrm{C}}$. Each boundary point $f(x)\left(x=e^{i \theta}\right)$ has either

(a) a local tangent of direction

$$
T(\theta)=\lim _{r \rightarrow 1} \arg \left(e^{i \theta} f^{\prime}\left(r e^{i \theta}\right)\right)+\frac{\pi}{2},
$$

which corresponds to the fact that $\mu(\{x\})=0$, 
or

(b) two local halftangents, so that $f(x)$ is a vertex of $\partial f(D)$ of an outer angle $2 \mu(\{x\}) \pi$, which corresponds to the fact that $\mu(\{x\}) \neq 0$.

In particular: $f$ has the angle property.

Moreover the images of the radial rays $f_{\theta}(r):=f\left(r e^{i \theta}\right)(r \in[0,1])$ divide the inner angle of $\partial f(D)$ at $f(x)$ in two equal parts.

Proof Let $f$ have boundary rotation $K \pi$. Then there is a Paatero representation (34). In this context it is more convenient to write (34) as a Stieltjes integral representation with the distribution function $m:[0,2 \pi] \rightarrow \mathrm{R}$ of $\mu$ defined by

$$
m(t):=\frac{1}{2}\left(\mu \left(\left[0, e^{i t}[)+\mu\left(\left[0, e^{i t}\right]\right)\right)+C,\right.\right.
$$

where $C \in \mathbf{R}$ is such that

$$
\int_{0}^{2 \pi}\left(m(t)-\frac{t}{2 \pi}\right) d t=0 .
$$

The Paatero representation (34) then reads

$$
\frac{f^{\prime \prime}}{f^{\prime}}(z)=-2 \int_{0}^{2 \pi} \frac{d m(t)}{z-e^{i t}}, \quad \int_{0}^{2 \pi} d m(t)=1, \quad \int_{0}^{2 \pi}|d m(t)|=\frac{K}{2} .
$$

Therefore it follows by integration (using the normalization $f^{\prime}(0)=1$ ) that

$$
\begin{aligned}
\ln f^{\prime}(z) & =\int_{0}^{z} \frac{f^{\prime \prime}}{f^{\prime}}(\zeta) d \zeta=-2 \int_{0}^{2 \pi} d m(t)\left(\int_{0}^{z} \frac{d \zeta}{\zeta-e^{i t}}\right) \\
& =-2 \int_{0}^{2 \pi} \ln \left(1-e^{-i t} z\right) d\left(m(t)-\frac{t}{2 \pi}\right),
\end{aligned}
$$

as

$$
\int_{0}^{2 \pi} \ln \left(1-e^{-i t} z\right) d t=0 \quad(z \in \mathrm{D})
$$

Observe that $m(t)-t /(2 \pi)$ is periodic with period $2 \pi$ by (38), so that an integration by parts gives with the aid of (37) that

$$
\begin{aligned}
\ln f^{\prime}(z) & =2 i \int_{0}^{2 \pi} \frac{e^{-i t} z}{1-e^{-i t} z}\left(m(t)-\frac{t}{2 \pi}\right) d t \\
& =i \int_{0}^{2 \pi} \frac{1+e^{-i t} z}{1-e^{-i t} z}\left(m(t)-\frac{t}{2 \pi}\right) d t
\end{aligned}
$$

from which it follows that

$$
\begin{aligned}
\arg f^{\prime}(z) & =\operatorname{Re} \int_{0}^{2 \pi} \frac{1+e^{-i t} z}{1-e^{-i t} z}\left(m(t)-\frac{t}{2 \pi}\right) d t \\
& =\int_{0}^{2 \pi} \frac{1-r^{2}}{1-2 r \cos (t-\theta)+r^{2}}\left(m(t)-\frac{t}{2 \pi}\right) d t
\end{aligned}
$$


By the definition (36) of $m$ it follows (see e.g. [10], p. 336) that $\left(z=r e^{i \theta}\right)$

$$
\lim _{r \rightarrow 1} \arg f^{\prime}(z)=2 \pi\left(m(\theta)-\frac{\theta}{2 \pi}\right),
$$

so that

$$
\lim _{r \rightarrow 1} \arg z f^{\prime}(z)=2 m(\theta) \pi
$$

This implies that

$$
N(\theta):=\lim _{r \rightarrow 1} \arg z f^{\prime}(z)
$$

exists for each $\theta \in[0,2 \pi]$ and is a function of bounded variation with

$$
N(\theta)=2 m(\theta) \pi, \quad \int_{0}^{2 \pi}|d N(\theta)|=\operatorname{br}(f)=K \pi .
$$

To get (a) and (b) we now use Paatero's result that $f$ has a continuous extension to $\bar{D}$, and that at each finite boundary point $f\left(e^{i \theta}\right)$ there is either a tangent to $\partial f(D)$ of direction $T(\theta)$ if $m$ is continuous at $\theta$, or two half-tangents of direction $T(\theta-0)$ and $T(\theta+0)$ such that $\partial f(D)$ has a vertex at $f\left(e^{i \theta}\right)$ whose outer angle equals the total jump of $m$ at $\theta$ (see [27], §7). An inspection of Paatero's proof shows that the same conclusion follows if $f(x)=\infty$, replacing the euclidean by the spherical distance and measuring angles and directions spherically.

Finally observe that $\arg z f^{\prime}(z)$ gives the normal direction of the level curve $f_{r}(\theta)$ $:=f\left(r e^{i \theta}\right)(\theta \in[0,2 \pi])$ at the point $z=r e^{i \theta}$, so that $\arg z f^{\prime}(z)+\pi / 2$ is the direction of the tangent. On the other hand the image of the radial ray $f_{\theta}$ cuts $f_{r}$ perpendicularly for all $r \in] 0,1[$ as $f$ is locally conformal. By (a) this remains true for $r=1$, if at $f(x)$ there exists a tangent, implying that $f_{\theta}$ divides the inner angle (namely $\pi$ ) in two equal parts. If $f(x)$ is a vertex of $\partial f(D)$, then by (36) the same conclusion follows.

Each signed measure $\mu$ on $\partial \mathrm{D}$ has a Lebesgue decomposition as the sum of some discrete, some continuously singular and some absolutely continuous part with respect to Lebesgue measure $\lambda_{1}$, i.e.

$$
\mu=\mu_{\mathrm{disc}}+\mu_{\mathrm{sing}}+\mu_{\mathrm{abs}},
$$

(see e.g. [24], p. 218, problem 4.3.12) where

$$
\mu_{\mathrm{disc}}=\sum_{k=1}^{\infty} \mu_{k} \delta_{x_{k}}
$$

( $\delta_{x}$ is the Dirac measure at $x$ ). We write $\mu_{\text {cont }}:=\mu_{\text {sing }}+\mu_{\text {abs }}$ for the continuous part of $\mu$.

The theorem has the consequence that

COROLLARY 2 Let $f \in V(K)$ such that the corresponding signed measure $\mu$ has a decomposition $\mu=\mu_{\mathrm{disc}}+\mu_{\mathrm{cont}}$. Then $\partial f(\mathrm{D})$ is smooth up to a countable number of vertices $f\left(x_{k}\right)(k \in N)$ of outer angles $2 \mu_{k} \pi$, say, and there is a one-io-one correspondence between those boundary points and $\mu_{\mathrm{disc}}$ such that (45) holds. 
For the largest and the smallest inner and outer angles $\alpha_{\max } \pi, \alpha_{\min } \pi, 2 \mu_{\max } \pi$ and $2 \mu_{\min } \pi$ il follows

$$
\begin{aligned}
\alpha_{\max } & =\max _{k \in N_{i}}\left|1-2 \mu_{k}\right|, \\
\alpha_{\min } & =\min _{k \in N_{0}}\left|1-2 \mu_{k}\right|, \\
2 \mu_{\max } & =2 \max _{k \in N}\left|\mu_{k}\right|, \\
2 \mu_{\min } & =0,
\end{aligned}
$$

where $\alpha_{0}:=1$.

Proof We have only to prove that the desired maxima and minima exist. But this follows easily as (a): $\mu_{k} \rightarrow 0$ for $k \rightarrow \infty$, and so 0 is the only cluster point of $\left\{l_{k}: k \in \mathbf{N}\right\}$, and (b): $\left\{\mu_{k}: k \in \mathbf{N}\right\}$ is bounded.

Part (b2) of the corollary is obviously equivalent to the existence of some smooth boundary point. The existence of the maxima and minima considered shows that $\alpha_{\text {iniax }} \pi, \alpha_{\text {inin }} \pi, 2 \mu_{\max } \pi$ and $2 \mu_{\text {inim }} \pi$ in fact represent the maximum and minimum of the inner and outer angles.

Now we are ready to generalize Theorem 1 to functions of bounded boundary rotation. Therefore we deduce the following formulas for the Koebe, Becker and Nehari expressions for functions of bounded boundary rotation with a representation (34) similar to $(30)-(33)$ : let

$$
b(z ; x):=\frac{1-\bar{z} x}{z-x}
$$

then

$$
\begin{aligned}
& \kappa(f ; z)=\left|\bar{z}+(1+r) \int_{\theta \mathbf{D}} \frac{1-r}{z-x} d \mu(x)\right|=\left|\int_{\theta \mathbf{D}} b(z ; x) d \mu(x)\right|, \\
& \lambda(f ; z)=2(1+r)\left|\int_{\theta \mathbf{D}} \frac{1-r}{z-x} d \mu(x)\right|,
\end{aligned}
$$

and, since

$$
\begin{aligned}
S_{f}(z) & =2 \int_{\partial \mathbf{D}} \frac{d \mu(x)}{(z-x)^{2}}-2\left(\int_{\partial \mathbf{D}} \frac{d \mu(x)}{z-x}\right)^{2} \\
& =\int_{(\partial \mathbf{D})^{2}} d \mu(x) d \mu(y)\left(\frac{1}{z-x}-\frac{1}{z-y}\right)^{2},
\end{aligned}
$$

we get

$$
\begin{aligned}
\sigma(f ; z) & =2(1+r)^{2}\left|\int_{\partial \mathbf{D}}\left(\frac{1-r}{z-x}\right)^{2} d \mu(x)-\left(\int_{\partial \mathbf{D}} \frac{1-r}{z-x} d \mu(x)\right)^{2}\right| \\
& =\left|\int_{(\delta \mathbf{D})^{2}} d \mu(x) d \mu(y)(b(z ; x)-b(z ; y))^{2}\right| .
\end{aligned}
$$


THEOREM 3 Let $f \in V(K)$ such that the corresponding signed measure $\mu$ has discrete part $\mu_{\mathrm{disc}}$ of form (45). Then

$$
\begin{aligned}
& \limsup _{z \rightarrow o \mathbf{D}} \kappa(f ; z)=\alpha_{\max }, \\
& \liminf _{z \rightarrow O \mathbf{D}} \kappa(f ; z)=\alpha_{\min }, \\
& \limsup _{z \rightarrow o \mathbf{D}} \lambda(f ; z)=4 \mu_{\max }, \\
& \limsup _{z \rightarrow O \mathbf{D}} \sigma(f ; z)=8 \max _{k \in \mathbb{N}}\left|\mu_{k}\left(1-\mu_{k}\right)\right| .
\end{aligned}
$$

Proof Let $f \in V(K)$ with corresponding signed measure $\mu$. As usual we write $\mu=\mu_{\mathrm{dis}}+\mu_{\mathrm{cont}}$ such that (45) holds. Then $\sum_{k=1}^{\infty}\left|\mu_{k}\right| \leq K / 2$. Let $\epsilon>0$ be given. Now choose $n \in \mathrm{N}$ large enough that

$$
\sum_{k=n+1}^{\infty}\left|\mu_{k}\right| \leq \epsilon
$$

and that the maximal value $\max _{k \in N}\left|\mu_{k}\right|=\left|\mu_{k_{0}}\right|$ is attained for $k_{0} \leq n$.

Let us first consider (b). The integral on the right hand side of (48) can be decomposed in three terms $\left(z=r e^{i \theta}\right)$

$$
\begin{aligned}
I(z) & =\int_{\partial \mathbf{D}} \frac{1-r}{z-x} d \mu(x) \\
& =\sum_{k=1}^{n} \mu_{k} \frac{1-r}{z-x_{k}}+\sum_{k=n+1}^{\infty} \mu_{k} \frac{1-r}{z-x_{k}}+\int_{\partial \mathbf{0}} \frac{1-r}{z-x} d \mu_{\text {cont }}(x) \\
& =I_{1}(z)+I_{2}(z)+I_{3}(z) .
\end{aligned}
$$

For $I_{1}$ we get by Lemma 4

$$
\lim _{r \rightarrow 1} I_{1}\left(r e^{i \theta}\right)=\left\{\begin{array}{cl}
-\mu_{k} \overline{x_{k}} & \text { if } \theta=\arg x_{k} \quad(k=1, \ldots, n) \\
0 & \text { otherwise }
\end{array},\right.
$$

so that

$$
\limsup _{z \rightarrow \delta \mathbf{D}}\left|I_{1}(z)\right|=\max _{1 \leq k \leq n}\left|\mu_{k}\right|=\max _{k \in \mathbb{N}}\left|\mu_{k}\right|=\mu_{\max }
$$

by the choice of $n$ and by Corollary 2 . Thus it remains to show that $I_{2}$ and $I_{3}$ tend to zero as $r$ tends to 1 . This follows for $I_{2}$ from (51) and for $I_{3}$ from the continuity of $\mu_{\text {cont }}$ (see e.g. [11]), which finishes the proof for (b). 
(a) As above we have a decomposition (see (47))

$$
\begin{aligned}
\kappa(f ; z) & =\left|\int_{\partial \mathbf{U}} \frac{1-\bar{z} x}{z-x} d \mu(x)\right| \\
& =\left|\sum_{k=1}^{n} \mu_{k} \frac{1-\bar{z} x_{k}}{z-x_{k}}+\sum_{k=n+1}^{\infty} \mu_{k} \frac{1-\bar{z} x_{k}}{z-x_{k}}+\int_{\partial \mathbf{O}} \frac{1-\bar{z} x}{z-x} d \mu_{\text {cont }}(x)\right| \\
& =\left|I_{1}(z)+I_{2}(z)+I_{3}(z)\right|
\end{aligned}
$$

for which we conclude

$$
\lim _{r \rightarrow 1} I_{1}\left(r e^{i \theta}\right)=\left\{\begin{array}{cl}
\overline{x_{k}}\left(1-2 \mu_{k}\right) & \text { if } \theta=\arg x_{k} \quad(k=1, \ldots, n) \\
1 & \text { otherwise }
\end{array}\right.
$$

and $\lim _{r \rightarrow 1} I_{2}\left(r e^{i \theta}\right)=\lim _{r \rightarrow 1} I_{3}\left(r e^{i \theta}\right)=0$, as $\left|b\left(z ; x_{k}\right)\right|$ is bounded by 1 for $z \in \mathbf{D}$ and $x_{k} \in \partial \mathbf{D}$.

(c) The same procedure shows that for $\lim _{r \rightarrow l} \sigma\left(f ; r e^{i \theta}\right)$ also the discrete part of $\mu$ is decisive.

As consequence we have

COROLlaRY 3 Let $f \in V(K)$. Then

$$
\underset{z \rightarrow O \mathbf{D}}{\limsup } \lambda(f ; z)=0 \Leftrightarrow f \quad \text { is bounded and } \partial f(\mathbf{D}) \text { is smooth. }
$$

Proof By Theorem 3 the left hand side is equivalent to $\mu_{\max }=0$, and this obviously is equivalent to the fact that $\mu_{\text {disc }}=0$, which by Corollary 2 is equivalent to the smoothness and boundedness of $\partial f(D)$.

\section{Moreover}

COROLLARY 4 Let $f \in V(K)$ such that the corresponding signed measure $\mu$ has discrete part $\mu_{\mathrm{disc}}$ of form (45). If further $\mu_{k} \geq-1 / 2(k \in N)$ (in particular, if $f$ is univalent), then

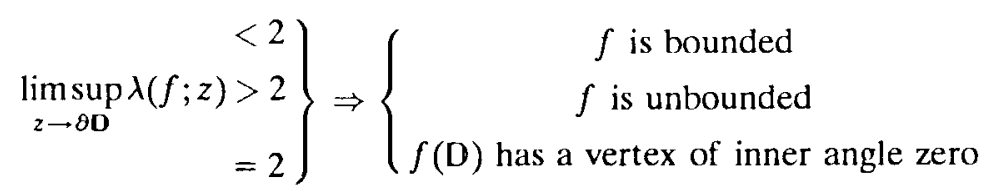

Proof By Theorem 3 the expression $\limsup _{z \rightarrow o \mathbf{D}} \lambda(f ; z)$ equals $4 \mu_{\max }$. Let now first this term be less or greater than 2 . Then by Theorem $2 f(D)$ has vertices of outer angles $2 \mu_{k} \pi(k \in N)$, and so is bounded and unbounded respectively by the definition of a vertex at $\infty$. On the other hand, if it equals 2 , then necessarily there is a vertex which corresponds to $\mu_{\max }=1 / 2$ of outer angle $\pi$, which gives the result. 
Becker ([3], p. 414) conjectured that for $f \in S$ with Jordan domain $f(D)$ the condition

$$
\limsup _{z \rightarrow \theta 0} \lambda(f ; z)<2
$$

implies that $f$ has a quasiconformal extension to $C$. This conjecture is true for functions of bounded boundary rotation.

COROLlary 5 Let $f \in S$ have bounded boundary rotation. Then (53) implies that $f$ has a quasiconformal extension to $\mathrm{C}$.

Proof Suppose $f \in V(K)$. As $f$ is univalent, by Corollary 4 condition (53) implies that $f$ is bounded. By Theorem 3 it follows moreover that $\mu_{\max }=: \frac{1}{2}(1-\epsilon)$ for some $\epsilon>0$. So for all vertices the relation $\left|1-\alpha_{k}\right| \leq 2 \mu_{\max }=1-\epsilon$ holds, and therefore $f \leq \alpha_{k} \leq 2-\epsilon(k \in N)$, so that there is a vertex of smallest angle $\alpha_{\min } \pi \geq \epsilon \pi$ and a vertex of largest angle $\alpha_{\max } \pi \leq(2-t) \pi$. Because $\mu_{k}, 0$ as $k \rightarrow \infty$ there are only a finite number of vertices with an outer angle near $t \pi$ (i.e. an inner angle $\alpha_{k} \pi \approx 0$ or $\left.\alpha_{k} \pi \approx 2 \pi\right)$, so that the local characterization of quasicircles due to Ahlfors ([1], see [22], chapter II, $\zeta 8$ ) shows that $\partial f(D)$ is a quasicircle.

Corollary 2 gives a one-to-one correspondence between the discrete part of the signed measure $\mu$ associated with $f$ and the vertices of $\partial f(D)$. Therefore it is of some interest to decide what kinds of boundary smoothness are typical for the parts of $\mu$ absolutely continuous and continuously singular with respect to Lebesgue measure. Here we get a partial result.

LEMMA 6 Let $f \in V(K)$ with $f(D)=F$ and $z_{0}=e^{i \theta_{0}}$ such that $f\left(z_{0}\right)$ is a point where the boundary curve $f\left(e^{i \theta}\right)$ is analytic. Then the function $m$ associated with $f$ by (38) is a $C^{\infty}$-function in a neighborhood of $\theta_{0}$.

Proof As $\partial F$ is analytic at $f\left(z_{0}\right)$ the Schwarz reflection principle shows that $f$ has an analytic extension at $z_{0}$. So in particular $f$ is analytic in a certain neighborhood $U$ of $z_{0}$ on the boundary of $\mathbf{D}$, and so is $f^{\prime}$. We deduce that moreover $f^{\prime}\left(z_{1}\right) \neq$ 0 for $z_{1}=e^{i \theta_{1}} \in U$. Suppose the contrary, then $f^{\prime}$ has an expansion $\left(\alpha_{1} \neq 0\right)$

$$
f^{\prime}(z)=\alpha_{1}\left(z-z_{1}\right)^{k}+\alpha_{2}\left(z-z_{1}\right)^{k+1}+\cdots
$$

for some $k \in \mathrm{N}$, which leads to

$$
\frac{f^{\prime \prime}}{f^{\prime}}(z)=\frac{k}{z-z_{1}}+H(z)
$$

with $H$ analytic in $U$. By the identity theorem for analytic functions (54) holds also in $\mathrm{D}$ so that by Theorem $2 \partial f(\mathrm{D})$ has a vertex at $f\left(z_{1}\right)$ (of outer angle $-k \pi$ ), in contrast to the analycity. Therefore $f^{\prime}\left(z_{1}\right) \neq 0$, and so $k=0$ in (54), i.e. $f^{\prime \prime} / f^{\prime}$ is analytic at $z_{1}$, and so is in $\left(f^{\prime}\right)$. In particular $\arg f^{\prime}\left(e^{i \theta}\right)$ is in $C^{\infty}$ at $\theta_{1}$ and so in $U$. By (42) the conclusion follows.

From this we get

THEOREM 4 Let $f \in V(K)$ with $f(D)=F$ such that $\partial F$ is analyic except at a countable number of points where of has a tangent. Then the signed measure $\mu$ associated with $f$ by (34) is absolutely continuous with respect to $\lambda^{1}$. 
Proof If $\partial F$ is analytic everywhere, then by the Lemma $m$ is in $C^{\infty}([0,2 \pi])$ and $d m=m^{\prime}(\theta) d \theta$, where $m^{\prime}$ in particular is integrable and its integral gives $m$, so $m$ and thus $\mu$ is absolutely continuous. If there is at most a countable number of points of nonanalycity on $\partial f(D)$, then-as there is no vertex- $m$ is the sum of the above constructed absolutely continuous part and some continuously singular part $m_{\text {sing }}$ with $m_{\text {sing }}^{\prime}=0$ a.e. Moreover $m_{\text {sing }}^{\prime}$ is continuous in $[0,2 \pi]$ except of some countable set $\Omega$ by the Lemma. So it has a unique continuous extension to $[0,2 \pi] \backslash \Omega$ which vanishes. Finally $\mu_{\text {sing }}$ must vanish as it is continuous in $[0,2 \pi]$ and its support $\Omega$ is countable.

Similarly one gets if there are vertices

THEOREM 5 Let $f \in V(K)$ with $f(\mathbf{D})=F$ such that $\partial F$ is analytic except of at most a countable number of points where $\partial F$ has a tangent and a countabie number of vertices $\boldsymbol{w}_{k}=f\left(x_{k}\right)$ of outer angle $2 \mu_{k} \pi(k \in \mathrm{N})$. Then the signed measure $\mu$ associated with f fulfills $\mu=\mu_{\mathrm{disc}}+\mu_{\mathrm{abs}}$ such that (45) holds.

\section{THE KOEBE, NEHARI AND BECKER EXPRESSIONS FOR CONVEX FUNCTIONS}

The results of the last section apply to convex functions. In this section we shall show that in the special case of convex functions also corresponding results for the terms $\sup _{z \in \mathbf{D}} \kappa(z)$, inf $f_{z \in \mathrm{D}} \kappa(z)$ and $\sup _{z \in \mathbf{D}} \sigma(z)$ are available. On the other hand our results give analytic representations for $\alpha_{\max }, \alpha_{\min }$ and $2 \mu_{\max }$. We remark that Pommerenke gave the following representation for the maximal outer angle

(see [28], Theorem 1).

$$
2 \mu_{\max }=\lim _{r \rightarrow 1} \frac{\ln \left(\max _{|z|=r}\left|f^{\prime}(z)\right|\right)}{\ln \frac{1}{1-r}} .
$$

THEOREM 6 Let $f \in K$, then

$$
\sup _{z \in \mathbf{D}} \kappa(f ; z)=\alpha_{\max }=1,
$$

in fact, (a1) is equivalent to the convexity of $f$.

$$
\underset{z \rightarrow O \mathbf{D}}{\limsup } \lambda(f ; z)=4 \mu_{\max }= \begin{cases}2\left(1-\alpha_{\min }\right) & \text { if } f \text { is bounded } \\ 2\left(1+\alpha_{\min }\right) & \text { if } f \text { is unbounded }\end{cases}
$$

(c1) $\quad \limsup _{z \rightarrow \partial \mathbf{D}} \sigma(f ; z)=8 \mu_{\max }\left(1-\mu_{\max }\right)=2\left(1-\alpha_{\min }^{2}\right)$.

If furthermore $f$ is unbounded, then

$$
\begin{aligned}
& \inf _{z \in \mathbf{D}} \kappa(f ; z)=\alpha_{\min }, \\
& \sup _{z \in \mathbf{D}} \sigma(f ; z)=2\left(1-\alpha_{\min }^{2}\right),
\end{aligned}
$$


and $\alpha_{\min } \pi$ is the angle of $\partial f(\mathrm{D})$ at $\infty$.

Proof

(a1) That this is equivalent to the convexity of $f$ follows from the fact that the universal linearly invariant family of order 1 is the family of convex functions (see [29], Folgerung 1.1 and Folgerung 2.4). On the other hand by Theorem 3 this is equivalent to the geometrical fact that all nonsmooth boundary points of $\partial f(D)$ have interior angles less than $\pi$ and the existence of some smooth boundary point.

(b) For convex functions and all $k \in N$ we have $\left.\mu_{k} \in\right] 0,1$, so that because of the relation $\alpha_{k}=\left|1-2 \mu_{k}\right|$ the value $\alpha_{k_{0}}=\alpha_{\min }$ is attained if the distance of $\mu_{k_{n}}$ and $1 / 2$ is minimal. If $f$ is unbounded, then $\mu_{\max }>1 / 2$, and this value is easily seen to minimize the distance to $1 / 2$. Otherwise also the largest value $\mu_{\max }<1 / 2$ minimizes this distance, so that finally

$$
\alpha_{\min }=\left|1-2 \mu_{\max }\right|,
$$

which leads to the result by Theorem 3 .

(c1) By Theorem 3 it follows that

$$
\limsup _{z \rightarrow O \mathbf{D}} \sigma(f ; z)=8 \max _{k \in \mathbf{N}}\left|\mu_{k}\left(1-\mu_{k}\right)\right| .
$$

As $\mu_{k}>0(k \in N)$ and because that value of $\left\{\mu_{k}\right\}$ nearest $1 / 2$ is $\mu_{\max }$ we see that this value maximizes the right hand side of (56) implying the result.

(a2) If $f$ is unbounded, then $\partial f(D)$ has a vertex at $\infty$ of angle $\alpha_{1} \pi=\alpha_{\min } \pi$ with corresponding outer angle $2 \mu_{1} \pi=2 \mu_{\max } \pi$.

Because $f(\mathbf{D})$ can be approximated by unbounded convex polygonal domains with fixed angle $\alpha_{1} \pi$ at $\infty$, it is sufficient to consider those SchwarzChristoffel mappings with $\mu_{1}=\left(1+\alpha_{1}\right) / 2$ and $\sum_{k=2}^{n} \mu_{k}=\left(1-\alpha_{1}\right) / 2$. Therefore we get with $(30)$ as $\left|b_{k}\right|=1(k=1, \ldots, n)$

$$
\begin{aligned}
\kappa(f ; z)=\left|\sum_{k=1}^{n} \mu_{k} b_{k}\right| & =\left|\mu_{1}+\sum_{k=2}^{n} \mu_{k} b_{k} \overline{b_{1}}\right| \\
& \geq \mu_{1}-\sum_{k=2}^{n} \mu_{k}=\frac{1+\alpha_{1}}{2}-\frac{1-\alpha_{1}}{2}=\alpha_{1} .
\end{aligned}
$$

Theorem 3 shows that $\liminf _{z \rightarrow o \mathbf{D}} \kappa(f ; z)=\alpha_{1}$, which gives the result.

(c2) Without loss of generality consider the same unbounded convex polygons with fixed angle $\alpha_{1} \pi$ at $\infty$. Then by (33) and (30) we get

$$
\begin{aligned}
\sigma(f ; z) & =\left|\sum_{j, k=1}^{n} \mu_{j} \mu_{k}\left(b_{j}(z)-b_{k}(z)\right)^{2}\right| \leq \sum_{j, k=1}^{n} \mu_{j} \mu_{k}\left|b_{j}(z)-b_{k}(z)\right|^{2} \\
& \leq 2\left(1-\left|\sum_{k=1}^{n} \mu_{k} b_{k}(z)\right|^{2}\right)=2\left(1-\kappa(f ; z)^{2}\right) \leq 2\left(1-\alpha_{\min }^{2}\right) .
\end{aligned}
$$


On the other hand by (c1) $\limsup _{z \rightarrow o \mathbf{D}} \sigma(f ; z)=2\left(1-\alpha_{\min }^{2}\right)$, which finishes the proof.

We remark that (c2) for unbounded convex functions in much stronger than the result given in [18], Theorem 3 , where the question was solved, which convex functions attain the maximal value 2 for the supremum of the Nehari expression.

We conjecture that the statement (c2) remains true if $f$ is bounded, because it seems to be true numerically. Moreover we conjecture that for bounded convex functions $\inf _{z \in \mathbf{D}} \kappa(f ; z)=0$.

The statement (a1) shows in particular that for the Koebe expression the sup and the lim sup coincide. We shall show in the sequel that for convex functions the Koebe expression satisfies moreover a certain maximum principle. Therefore we need the

LEMMA 7 Let $f(z)=z+a_{2} z^{2}+a_{3} z^{3}+\cdots$ be locally univalent. If the Koebe expression $n(f ; z)$ has a local maximum at $z_{0}=0$, then

$$
a_{3}=\frac{1}{3 \mid a_{2}^{2}}\left(1+2\left|a_{2}\right|^{2}\right),
$$

in particular

$$
\left|3 a_{3}-2 a_{2}^{2}\right|=1
$$

Proof Let

$$
F(r, \theta):=r e^{-i \theta}-\frac{1}{2}\left(1-r^{2}\right) \frac{f^{\prime \prime}}{f^{\prime}}\left(r e^{i \theta}\right)
$$

and

$$
G(r, \theta):=F(r, \theta) \cdot \overline{F(r, \theta)}=\kappa\left(f ; r e^{i \theta}\right)^{2},
$$

then for a local maximum of $\kappa(f ; z)$ at the origin obviously

$$
\left.\frac{\partial G}{\partial r}\right|_{r=0}=0
$$

holds for all $\theta \in \mathbf{R}$. From

$$
\frac{\partial G}{\partial r}=2 \cdot \operatorname{Re}\left(\frac{\partial F}{\partial r} \cdot \bar{F}\right)
$$

and

$$
\frac{\partial F}{\partial r}(r, \theta)=e^{-i \theta}-\frac{1}{2}\left(1-r^{2}\right) e^{i \theta}\left(\frac{f^{\prime \prime}}{f^{\prime}}\right)^{\prime}\left(r e^{i \theta}\right)+r \frac{f^{\prime \prime}}{f^{\prime}}\left(r e^{i \theta}\right)
$$

we get therefore for all $\theta \in \mathbf{R}$ the relation

$$
\operatorname{Re}\left\{\left(e^{-i \theta}-\frac{1}{2} e^{i \theta}\left(\frac{f^{\prime \prime}}{f^{\prime}}\right)^{\prime}(0)\right) \overline{\left(\frac{f^{\prime \prime}}{f^{\prime}}(0)\right)}\right\}=0 .
$$


This implies either $a_{2}=0$-which leads to a local minimum of $\kappa(f ; z)$ at the originor, using the notations $(\rho>0)$

$$
2 \bar{a}_{2}=\overline{\left(\frac{f^{\prime \prime}}{f^{\prime}}(0)\right)}=: \rho e^{i p}
$$

and

$$
3 a_{3}-2 a_{2}^{2}=\frac{1}{2}\left(\frac{f^{\prime \prime}}{f^{\prime}}\right)^{\prime}(0)=: x+i y=b,
$$

we get for all $\theta \in \mathbf{R}$

$$
\begin{aligned}
& \operatorname{Re}\{(\cos \theta-i \sin \theta-(\cos \theta+i \sin \theta)(x+i y)) \rho(\cos \varphi+i \sin \varphi)\} \\
& \quad=\rho \cdot \operatorname{Re}\{((1-x) \cos \theta+y \sin \theta-i((1+x) \sin \theta+y \cos \theta))(\cos \varphi+i \sin \varphi)\} \\
& \quad=\rho(\cos \theta[(1-x) \cos \varphi+y \sin \varphi]+\sin \theta[y \cos \varphi+(1+x) \sin \varphi])=0,
\end{aligned}
$$

so that the coefficients of the terms $\cos \theta$ and $\sin \theta$ must vanish. This implies the relations

$$
\begin{aligned}
& (1-x) \cos \varphi=y \sin \varphi, \\
& (1+x) \sin \varphi=-y \cos \varphi,
\end{aligned}
$$

from which we deduce by multiplication that

$$
|b|^{2}=x^{2}+y^{2}=1,
$$

and so (58). Now we substitute $b=x+i y=: e^{i \beta}$ into (61) and (62), and a short calculation gives the two equations

$$
\begin{aligned}
& \cos (\varphi+\beta)=\cos \varphi, \\
& \sin (\varphi+\beta)=-\sin \varphi,
\end{aligned}
$$

which finally lead to the unique solution $\beta=-2 \varphi$ implying the result.

The next lemma shows that only every special convex functions satisfy (58).

LEMMA 8 Let $f \in K$. Then relation (58) implies that

$$
\frac{f^{\prime \prime}}{f^{\prime}}(z)=-2 \frac{t}{z-x}-2 \frac{1-t}{z+x}
$$

for some $t \in[0,1]$ and some $x \in \partial \mathrm{D}$, in particular: $f(\mathrm{D})$ is either a half-plane, a sector or a parallel strip.

Proof If $f(z)=z+a_{2} z^{2}+a_{3} z^{3}+\cdots \in K$, then $p(z)=1+z\left(f^{\prime \prime} / f^{\prime}\right)(z)=$ $1+p_{1} z+p_{2} z^{2}+\cdots \in P$. So

$$
\left|p_{2}\right|=\left|6 a_{3}-4 a_{2}^{2}\right| \leq 2
$$

with equality if and only if

$$
p(z)=t\left(\frac{1+\bar{x} z}{1-\bar{x} z}\right)+(1-t)\left(\frac{1-\bar{x} z}{1+\bar{x} z}\right)
$$


for some $t \in[0,1]$ and some $x \in \partial D$ (see e.g. [32], Corollary 2.3). This gives the result.

Now we have

THEOREM 7 Let $f \in K$. Then the Koche expression $r(f ; z)$ satisfies a maximum principle, i.e. it lakes its maximum over each domain $D$ which is properly contained in $\mathrm{D}$ (such that its closure lies in $\mathrm{D}, \mathrm{too})$ at the boundary of $\mathrm{D}$. In particular: the function

$$
K(r):=\sup _{|z|=r}\left|-\bar{z}+\frac{1}{2}\left(1-|z|^{2}\right) \frac{f^{\prime \prime}}{f^{\prime}}(z)\right|
$$

is monotonically increasing for $r \in[0,1]$.

Proof We shall prove that for $f \in K$ a local maximum of the expression $\kappa(f: z)$ can only occur at $\mathbf{a}_{\text {point }} z_{0} \in \mathrm{D}$ if $f(D)$ is either a half-plane or a sector, and in those cases the extremal value is attained at a curve joining $z_{0}$ with the boundary, namcly at a Steiner curcle, i.e. the image of the segment ]-1,1[ under an automorphism of $\mathrm{D}$, which gives the resuit.

Suppose first that $\kappa(f ; z)$ has a local maximum at 0 . Then by Lemma 7 (58) holds and by Lemma $8 f$ is of form (65). From this representation one deduces that

$$
z-\frac{1}{2}\left(1-r^{2}\right) \frac{f^{\prime \prime}}{f^{\prime}}(z)=\bar{z}+\frac{1-r^{2}}{z^{2}-x^{2}}(t(z+x)+(1-t)(z-x)),
$$

and especially for $z:=r x(r \in]-1,1[)$ it follows that

$$
\bar{z}-\frac{1}{2}\left(1-r^{2}\right) \frac{f^{\prime \prime}}{f^{\prime}}(z)=(1-2 t) \bar{x} .
$$

So $s(f ; z)$ is constant on some diameter of $D$, which was to prove. In the case of a parallel strip $(t=1 / 2)$ the extremal value of $\kappa(f ; z)$ obviously is a minimum, so that this case must not be considered.

On the other hand, if $\kappa(f ; z)$ has a local maximum at a point $z_{0} \neq 0$, then by Lemma 1 the information which we deduced at the origin can be transformed by an automorphism $\omega$ of $\mathrm{D}$, as the family $K$ of convex functions is linearly invariant. This gives the result.

\section{CONVEX FUNCTIONS WITH VANISHING SECOND COEFFICIENT}

Suppose, $f_{m}$ has the special form $(m \subset N)$

$$
f_{m}(z)=z+a_{m+1} z^{m+1}+a_{m+2} z^{m+2}+\cdots,
$$

then $f_{m} \rightarrow z$ as $m \rightarrow \infty$. Hence $f_{m}(\mathrm{D})$ tends to a disk in the sense of Carathéodory kernel convergence (if $f_{m}$ are univalent). So it seems to be plausible that the geometry of $f_{m}(D)$ will be restricted in some sense in connection with the restriction of some analytic properties. 
The next theorem gives a sharp version of these considerations in the case of convex functions. Therefore we need the

LEMMA 9 Let $f \prec g$ and $r \in] 0,1]$. Then

$$
\sup _{z \in \mathbf{D}_{r}}\left(1-|z|^{2}\right)\left|f^{\prime}(z)\right| \leq \sup _{z \in \mathbf{D}_{r}}\left(1-|z|^{2}\right)\left|g^{\prime}(z)\right|
$$

(see e.g. [32], p. 35, formula (4)).

THEOREM 8 Let $m \in N$ and $f_{m} \in K$ of form (66). Then $\lambda\left(f_{m} ; z\right) \leq 4 / m$, and this result is sharp for the function $G_{m}$ with

$$
G_{m}^{\prime}(z)=\frac{1}{\left(1-z^{m}\right)^{2 / m}}, \quad G(0)=0 .
$$

By Theorem 6 this has the geometric consequence that for $\int_{m}(\mathrm{D})$ hold

(a) $2 \mu_{\max } \pi \leq(1 / m) 2 \pi$,

(b) for $m \geq 2$ moreover $\alpha_{\min } \pi \geq(1-2 / m) \pi$,

(c) and for $m \geq 3 f_{m}$ is bounded.

Proof For a convex function of the given form it is well-known that

$$
f_{m}^{\prime} \prec \frac{1}{(1-z)^{2 / m}}
$$

(see e.g. [9]). This statement is equivalent to $\ln f_{m}^{\prime} \prec \ln h^{\prime}:=-(2 / m) \ln (1-z)$, so that by the lemma we only have to observe that

$$
\sup _{z \in \mathbf{D}}\left(1-|z|^{2}\right)\left|\frac{h^{\prime \prime}}{h^{\prime}}(z)\right|=\sup _{z \in \mathbf{D}} \frac{2}{m}\left(1-|z|^{2}\right) \frac{1}{1-|z|}=\frac{4}{m} .
$$

For the function $G_{m}$, defined by (67), one gets, choosing $z=r>0$, that

$$
\left(1-|z|^{2}\right)\left|\frac{G_{m}^{\prime \prime}}{G_{m}^{\prime}}(z)\right|=\left(1-r^{2}\right) \frac{2 \cdot r^{m-1}}{1-r^{m}}=(1+r) \frac{2 \cdot r^{m-1}}{1+r+\cdots+r^{m-1}} \stackrel{(r \rightarrow 1)}{\longrightarrow} \frac{4}{m},
$$

which establishes the statement about equality.

We remark that the statements (b) and (c) are obvious geometrical facts for $m$ fold symmetric convex functions, and the theorem generalizes these facts.

For convex functions with vanishing second coefficient we have as a

COROLlaRY 6 Let $f \in K$ with $a_{2}(f)=0$. Then either $f$ is bounded or $f$ is unbounded and has a zero angle at $\infty$. 
Proof Applying the theorem for $m=2$ we get $\mu_{\max } \leq 1 / 2$. By the geometrical interpretation as outer angle the result follows.

Finally we have the

COROLlaRY 7 Let $f(z)=z+a_{2} z^{2}+a_{3} z^{3}+\cdots \in K$ with $a_{2}=a_{3}=a_{4}=0$. Then $f$ fulfills the Becker univalence criterion.

\section{CONVEX FUNCTIONS WITH ANGLE $\alpha \pi$ AT $\infty$}

In Corollary 6 geometrical conditions had been given for $f \in K$ with $a_{2}(f)=0$ : either $f$ is bounded or $f$ is unbounded and $f(D)$ has a zero angle at $\infty$.

In this section we consider unbounded convex functions with given angle at $\infty$ and get results in the opposite direction.

For $\alpha \in[0,1]$ let $K(\alpha) \subset K$ denote the family of unbounded convex functions with inner angle $\alpha \pi$ at $\infty$. Obviously $K(1)$ consists only of half-plane mappings, so

$$
K(1)=\left\{f \in K \mid f(z)=\frac{z}{1-x z}, x \in \partial \mathbf{D}\right\} .
$$

The family $K(\alpha)$ is a linearly invariant family of order 1 .

The compactness of $K$ shows that if $\alpha \rightarrow 1$ then $f_{\alpha} \in K(\alpha)$ implies that $f_{\alpha} \rightarrow f \in$ $K(1)$, and so $\left|a_{n}\left(f_{\alpha}\right)\right| \rightarrow 1$ for all $n \in \mathrm{N}$. The following theorem gives more detailed information for the second and third coefficients.

THEOREM 9 Let $\alpha \in[0,1]$ and $f \in K(\alpha)$. Then

(a) $\left|a_{2}(f)\right| \geq \inf _{z \in \mathbf{D}} \kappa(f ; z)=\alpha$,

(b) $\limsup _{z \rightarrow \partial \mathbf{D}} \lambda(f ; z)=2(1+\alpha)$,

(c) $6\left|a_{3}(f)-a_{2}^{2}(f)\right| \leq \sup _{z \in \mathbf{D}} \sigma(f ; z)=2\left(1-\alpha^{2}\right)$.

If $\tau(f ; a):=\left|a_{3}\left((f \circ \omega-f \circ \omega(0)) /(f \circ \omega)^{\prime}(0)\right)\right|, \omega(z)=(z+a) /(1+\bar{a} z)$ and $\mu=$ $(1+\alpha) / 2$, then for $\alpha>1 / 2(\sqrt{13}-3)=0.3027 \ldots$ furthermore

(d) $\left|a_{3}(f)\right| \geq \inf _{z \in \mathbf{D}} \tau(f ; z) \geq \frac{1}{3}\left(4 \mu^{2}+2 \mu-3\right)=\frac{1}{3}\left(\alpha^{2}+3 \alpha-1\right)$.

For all $f \in K$ holds

(e) $\liminf _{z \rightarrow O \mathbf{D}} \tau(f ; z)=\frac{1}{3}\left(3-8 \mu_{\max }+8 \mu_{\max }^{2}\right)$,

in particular for $f \in K(\alpha)$

(f) $\liminf _{z \rightarrow o \mathbf{D}} \tau(f ; z)=\frac{1}{3}\left(3-8 \mu+8 \mu^{2}\right)=\frac{1}{3}\left(1+2 \alpha^{2}\right)$.

Proof The statements (a), (b) and (c) are obvious consequences of Theorem 6. Let us now consider the absolute value $\tau(f ; a)$ of the third coefficient of the Koebe transform $h:=(f \circ \omega-f \circ \omega(0)) /(f \circ \omega)^{\prime}(0)$. If $f$ is a polygonal function with an angle $\alpha \pi$ at $\infty$ then without loss of generality $\mu_{1}=\mu$, and so $\sum_{k=2}^{n} \mu_{k}=1-\mu$. By 
(29)-(33), and as $\left|b_{k}(a)\right| \leq 1(k=1, \ldots, n)$, we have

$$
\begin{aligned}
\tau(f ; a) & =\frac{\left|h^{\prime \prime \prime}(0)\right|}{6} \\
& =\frac{1}{6} \mid\left(1-\frac{\left.|a|^{2}\right)^{3} f^{\prime \prime \prime}(a)-6 \bar{a}\left(1-|a|^{2}\right)^{2} f^{\prime \prime}(a)+6 \bar{a}^{2}\left(1-|a|^{2}\right) f^{\prime}(a)}{\left(1-|a|^{2}\right) f^{\prime}(a)} \mid\right. \\
& =\left|\frac{1}{6}\left(1-|a|^{2}\right)^{2} S_{f}(a)+\left(-\bar{a}+\frac{1}{2}\left(1-|a|^{2}\right) \frac{f^{\prime \prime}}{f^{\prime}}(a)\right)^{2}\right| \\
& =\left|\frac{1}{6} \sum_{j \cdot k=1}^{n} \mu_{j} \mu_{k}\left(b_{j}(a)-b_{k}(a)\right)^{2}+\left(\sum_{k=1}^{n} \mu_{k} b_{k}(a)\right)^{2}\right| \\
& =\frac{1}{3}\left|\sum_{k-1}^{n} \mu_{k} h_{k}(a)^{2}+2\left(\sum_{k=1}^{n} \mu_{k} b_{k}(a)\right)^{2}\right| \\
& =\frac{1}{3}\left|\mu+\sum_{k=2}^{n} \mu_{k} b_{k}^{2} \bar{b}_{1}^{2}+2\left(\mu^{2}+2 \mu \sum_{k=2}^{n} \mu_{k} b_{k} \bar{b}_{1}+\left(\sum_{k=2}^{n} \mu_{k} b_{k} \bar{b}_{1}\right)^{2}\right)\right| \\
& \geq \frac{1}{3}\left(\mu+2 \mu^{2}-(1-\mu)-4 \mu(1-\mu)-2(1-\mu)^{2}\right) \\
& =\frac{1}{3}\left(4 \mu^{2}+2 \mu-3\right)=\frac{1}{3}\left(\alpha^{2}+3 \alpha-1\right)
\end{aligned}
$$

which gives the result by approximation.

(e), (f): This is proved in a way similar to the proof of Theorem 3.

We remark that the right hand side of inequality (d) tends to 1 as $\alpha \rightarrow 1$, and so gives a rather sharp estimate for values of $\alpha$ near 1 . The statement (a) shows that $K(\alpha)$ is an example of a linearly invariant family for which inf $f \in K(\alpha)\left|a_{2}(f)\right|$ is bounded from below.

\section{THE KOEBE, NEHARI AND BECKER EXPRESSIONS FOR CLOSE-TO-CONVEX FUNCTIONS}

By $C_{m}(\beta)$ we denote the family of $m$-fold symmetric close-to-convex functions of order $\beta$. It is easy to see using the original development [14] that the corresponding function $p \in \tilde{P}$ is of the special form

$$
p(z)=1+c_{m} z^{m}+c_{2 m} z^{2 m}+\cdots .
$$

For to consider those functions we need the following LEMMA 10 Let $x \in \partial \mathrm{D}, \lambda \in \mathbf{R}^{+}$and $h^{\prime} \prec((1+x z) /(1-z))^{\lambda / 2}$. Then $\lambda(h ; z) \leq \lambda$. 
Proof As we have $\ln h^{\prime} \prec \lambda / 2 \ln ((1+x z) /(1-z))$, Lemma 9 implies that

$$
\sup _{z \in \mathbf{D},}\left(1-|z|^{2}\right)\left|\frac{h^{\prime \prime}}{h^{\prime}}(z)\right| \leq \frac{|1+x|}{2} \lambda
$$

and so the result follows.

Now we have

THEOREM 10 L't $m \in N, \beta>0$ and $f(z)=z+a_{m+1} z^{m+1}+a_{2 m+1} z^{2 m+1}+\cdots$ an $m$-fold symmetric close-to-convex function of order $\beta$. Then $\lambda(f ; z) \leq(4 / m)+2 \beta$, and this is sharp for the function $F_{m}$ given by

$$
F_{m}^{\prime}(z)=\frac{\left(1+z^{m}\right)^{j}}{\left(1-z^{m}\right)^{\beta+2 / m}}, \quad F_{m}(0)=0
$$

Proof Let $f$ have the properties considered. Then there is an $m$-fold symmetric convex function $g$, a complex number $x \in \partial \mathbf{D}$ and a function $p \prec(1+x z) /(1-z)$ such that $f^{\prime}=g^{\prime} \cdot p^{\beta}$. Thus we have by Theorem 8 and Lemma 10 with $p:=h^{\prime}$

$$
\begin{aligned}
\sup _{z \in \mathbf{D}}\left(1-|z|^{2}\right)\left|\frac{f^{\prime \prime}}{f^{\prime}}(z)\right| & \leq \sup _{z \in \mathbf{D}}\left(1-|z|^{2}\right)\left|\frac{g^{\prime \prime}}{g^{\prime}}(z)\right|+\beta \sup _{z \in \mathbf{D}}\left(1-|z|^{2}\right)\left|\frac{p^{\prime}}{\vec{p}}(z)\right| \\
& \leq \frac{4}{m}+2 \beta
\end{aligned}
$$

For the function $F_{m}$, defined by (70), one gets, choosing $z=r>0$, that

$$
\left(1-|z|^{2}\right)\left|\frac{F_{m}^{\prime \prime}}{F_{m}^{\prime}}(z)\right|=\left(1-r^{2}\right) \frac{2 \cdot r^{m-1}}{1-r^{m}}+\beta\left(1-r^{2}\right) \frac{2 m \cdot r^{m-1}}{1-r^{2 m}} \stackrel{(r \rightarrow 1)}{\longrightarrow} \frac{4}{m}+2 \beta,
$$

which establishes the statement about equality.

We remark that for $m=1$ the statement is an immediate consequence of the linearly invariance of $C(\beta)$ because for $f \in C(\beta)$ one has $k(f ; z) \leq 1+\beta$ (see e.g. [29], Lemma 1.2), implying that

$$
\left(1-|z|^{2}\right)\left|\frac{f^{\prime \prime}}{f^{\prime}}(z)\right| \leq 2\left|-\bar{z}+\frac{1}{2}\left(1-|z|^{\overline{2}}\right) \frac{f^{\prime \prime}}{f^{\prime}}(z)\right|+2|z| \leq 4+2 \beta .
$$

The theorem gives

COROLlaRY 8 Let $\beta<1 / 2, m \geq 4 /(1-2 \beta)$ and $f \in C_{m}(\beta)$. Then $f$ fulfills the Becker univalence criterion. 


\section{INTEGRAL MEANS}

For $f \in A$ and $r \in[0,1[$ let

$$
\begin{aligned}
& M_{p}(r, f):=\left(\frac{1}{2 \pi} \int_{0}^{2 \pi}\left|f\left(r e^{i \theta}\right)\right|^{p} d \theta\right)^{1 / p} \quad(p \in] 0, \infty[), \\
& M_{\infty}(r, f):=\max _{\theta \in[0,2 \pi]}\left|f\left(r e^{i \theta}\right)\right|
\end{aligned}
$$

denote the $p$ th integral means. For $p \in] 0, \infty]$ let $H^{p}$ denote the family of functions $f$ for which $M_{p}(r, f)$ remains bounded as $r \rightarrow 1$.

$M_{p}(r, f)$ turns out to be a nondecreasing function of $r$ and also nondecreasing as function of $p$. For $f \in H^{p}$ the radial limit

$$
f\left(e^{i \theta}\right):=\lim _{r \rightarrow 1} f\left(r e^{i \theta}\right)
$$

turns out to exist for almost all $\theta \in[0,2 \pi]$ and is in $L^{P}([0,2 \pi])$, and

$$
M_{p}(1, f):=\left(\frac{1}{2 \pi} \int_{0}^{2 \pi}\left|f\left(e^{i \theta}\right)\right|^{p} d \theta\right)^{1 / \rho}=\lim _{r \rightarrow 1} M_{p}(r, f) .
$$

The Littlewood subordination theorem states that $f \prec F$ implies that $M_{p}(r, f) \leq$ $M_{p}(r, F)$ for all $\left.\left.p \in\right] 0, \infty\right]$ and all $r \in[0,1]$.

If the derivative $f^{\prime}$ of some function $f \in A$ is in $H^{p}$ for some $\left.p \in\right] 0, \infty$ ], then so is $f$, i.e.

$$
f^{\prime} \in H^{p} \Rightarrow \begin{cases}f \in H^{\infty} & \text { if } \quad p \geq 1 \\ f \in H^{p /(1-p)} & \text { otherwise }\end{cases}
$$

Moreover if $\int \in S$ maps $D$ onto some bounded Jordan domain, then

$$
f^{\prime} \in H^{1} \Leftrightarrow \partial f(D) \text { is rectifiable. }
$$

For functions $f$ which are in $H^{p}$ for some $\left.\left.p \in\right] 0, \infty\right]$ we define the Hardy-dimension of $f$ by

$$
\left.\left.\operatorname{dim}_{H P}(f):=\sup \{p \in] 0, \infty\right] \mid f \in H^{P}\right\} .
$$

(References: [23], [5].)

\section{INTEGRAL MEANS FOR POLYGGONS}

If $f$ is a polygonal mapping normalized by (1), then by the Schwarz-Christoffel formula (8) one has

$$
f^{\prime}(z)=\prod_{k=1}^{n} \frac{1}{\left(1-\overline{x_{k}} z\right)^{2 \mu_{k}}}, \quad x_{k} \in \partial \mathrm{D} \quad(k=1, \ldots, n), \quad \sum_{k=1}^{n} \mu_{k}=1 .
$$


From this representation one can see at once that $f^{\prime} \in H^{P}$ for some $p>0$ (namely for all $p<1 / 2$, sec e.g. [12], p. 80), so that $f^{\prime}\left(e^{i \theta}\right)$ exists for almost all $\theta \in[0,2 \pi]$ and

$$
\lim _{r \cdot 1} \int_{0}^{2 \pi}\left|f^{\prime}\left(r e^{i \theta}\right)\right|^{P} d \theta=\int_{0}^{2 \pi}\left|f^{\prime}\left(e^{i \theta}\right)\right|^{P} d \theta .
$$

For to get a sharp $H^{P}$-result for a polygonal mapping $f$ depending only on the parameters of the Schwarz-Christoffel formula, hence on the geometry of the image surface of $f$, we assume without loss of generality that $\mu_{k}>0(k=1, \ldots, m)$ and $\nu_{k}:=-\mu_{k+m}>0(k=1, \ldots, n-m)$ and write $y_{k}:=x_{k+m}(k=1, \ldots, n-m)$. Then

$$
f^{\prime}(z)=\prod_{k=1}^{n} \frac{1}{\left(1-\overline{x_{k}} z\right)^{2 \mu_{k}}}=\frac{\prod_{k=1}^{n-m}\left(1-\overline{y_{k}} z\right)^{2 \nu_{k}}}{\prod_{k=1}^{m}\left(1-\overline{x_{k}} z\right)^{2 \mu_{k}}},
$$

and so

$$
\left|f^{\prime}(z)\right|<\frac{\prod_{k=1}^{n-m} 2^{2 x_{k}}}{\prod_{k=1}^{m}\left|1 \quad x_{k} z\right|^{2 \mu_{k}}} .
$$

As $\sum_{k=1}^{n m} \nu_{k}<\sum_{k=1}^{n}\left|\mu_{k}\right|=: K / 2$ we get

$$
\left|f^{\prime}(z)\right|<2^{K} \frac{1}{\prod_{k=1}^{m}\left|1-\overline{x_{k}} z\right|^{2 \mu_{k}}} .
$$

By (75) we have to check the finiteness of

$$
\int_{0}^{2 \pi} \frac{d \theta}{\prod_{k-1}^{m}\left|1-\overline{x_{k}} e^{i \theta}\right|^{2 \mu_{k} P}} .
$$

Therefore suppose without loss of generality that $x_{k}(k=1, \ldots, m)$ are ordered successively on $\partial \mathrm{D}$ and define $\left(x_{m+1}:=x_{1}\right)$

$$
d:=\min \left\{\operatorname{dist}\left(x_{k}, x_{k+1}\right) \mid k=1, \ldots, m\right\} .
$$

Clearly $d>0$ as the points $x_{k}(k=1, \ldots, m)$ are isolated. (On the other hand the value of $d$ depends heavily on $n$ and for all sequences $\left(x_{k}\right)_{k \in N}$ of unimodular numbers $d \rightarrow 0$ as $n \rightarrow \infty$.) Now we decompose the integral (77) in $m$ components. Choose $t_{k}:=\frac{1}{2}\left(\arg \left(x_{k}\right)+\arg \left(x_{k-1}\right)\right)(k=1, \ldots, m)$, and observe that

$$
\left|1-\overline{x_{k}} e^{i \theta}\right|=\left|e^{i \theta}-x_{k}\right|>\frac{d}{2} \quad(k=1, \ldots, m)
$$

for $\theta \notin\left[t_{k-1}, t_{k}\right],\left(t_{m+1}:=t_{1}\right)$. Now it follows for $j=1, \ldots, m$ that

$$
\begin{aligned}
\int_{t_{j}}^{t_{j+1}} \frac{d \theta}{\prod_{k=1}^{m}\left|1-\overline{x_{k}} e^{i \theta}\right|^{2 \mu_{k} p}} & <\left(\frac{2}{d}\right)^{K p} \cdot \int_{t_{j}}^{t_{j+1}} \frac{d \theta}{\left|1-\overline{x_{j}} e^{i \theta}\right|^{2 \mu_{j} p}} \\
& \leq\left(\frac{2}{d}\right)^{K p} \cdot \int_{0}^{2 \pi} \frac{d \theta}{\left|1-\overline{x_{j}} e^{i \theta}\right|^{2 \mu, p}},
\end{aligned}
$$


which is finite if and only if $p<1 /\left(2 \mu_{j}\right)$. So (77) is finite iff $p<1 /\left(2 \mu_{\max }^{+}\right)$, where $\mu_{\text {max }}^{+}=\max \left\{\mu_{k} \mid k=1, \ldots, n\right\}$. This gives

LEMMA 11 Le't $f$ be a Schwar-Christoffel mapping. Then $f^{\prime} \in H^{p}$ for all $p<$ $1 /\left(2 \mu_{\max }^{+}\right)$, and this bound is sharp, i.e.

$$
\operatorname{dim}_{l^{r}}\left(f^{\prime}\right)=\frac{1}{2 \mu_{\max }^{+}}
$$

Analogously one gets for $1 / f^{\prime}$

LEMMA 12 Let $f$ he a Schwarz-Christoffel mapping. Then $1 / f^{\prime} \in H^{p}$ for all $p<$ $1 /\left(\alpha_{\max }-1\right)$, and this bound is sharp, i.e.

$$
\operatorname{dim}_{H^{p}}\left(1 / f^{\prime}\right)=\frac{1}{\alpha_{\max }-1}
$$

Proof The same procedure as above shows that $1 / f^{\prime} \in H^{p}$ for all $p<1 /\left(2 l_{\max }\right)$ where $\nu_{\text {max }}:=\max \left\{u_{k} \mid k=1, \ldots, n \quad m\right\}$. By (9) it follows that $2 \nu_{\text {max }}^{\prime}=-\left(1-\alpha_{\max }\right)$.

\section{INTEGRAL MEANS FOR FUNCTIONS OF BOUNDED BOUNDARY ROTATION}

For functions of bounded rotation $K \pi$ we have the usual representation (34)

$$
\frac{f^{\prime \prime}}{f^{\prime}}(z)=-2 \int_{0 \mathrm{D}} \frac{d \mu(x)}{z-x}
$$

for some signed measure $\mu$ with Lebesgue decomposition $\mu=\mu_{\text {disc }}+\mu_{\text {cont }}$. Then $\mu_{\text {disc }}=\sum_{k=1}^{x} \mu_{k} \partial_{x_{k}}$ for $x_{k} \in O D(k \in N)$ and $\sum_{k=1}^{\infty}\left|\mu_{k}\right| \leq K / 2$. Let now $\epsilon>0$ be given and choose $m \in N$ large enough that

$$
\sum_{k=m+1}^{\infty}\left|\mu_{k}\right| \leq \epsilon
$$

and that the maximal value $\mu_{\max }=\left|\mu_{k_{0}}\right|$ is attained for $k_{0} \leq m$. We write $y_{k}:=x_{k}$, $(k>m)$ and get

$$
\frac{f^{\prime \prime}}{f^{\prime}}(z)=-2 \sum_{k=1}^{m} \frac{\mu_{k}}{z-x_{k}}-2 \sum_{k=m+1}^{\infty} \frac{\mu_{k}}{z-y_{k}}-2 \int_{\partial \mathbf{D}} \frac{d \mu_{\mathrm{cont}}(x)}{z-x} .
$$

For the last expression we write

$$
-2 \int_{O \mathbf{D}} \frac{d \mu_{\mathrm{cont}}(x)}{z-x}=: \frac{k^{\prime \prime}}{k^{\prime}}(z)
$$

so that an integration gives (without loss of generality $f$ is always assumed to be normalized by (1)),

$$
f^{\prime}(z)=\prod_{k=1}^{n} \frac{1}{\left(1-\overline{x_{k}} z\right)^{2 \mu_{k}}} \cdot \prod_{k=m+1}^{\infty} \frac{1}{\left(1-\overline{y_{k}} z\right)^{2 \mu_{k}}} \cdot k^{\prime}(z)
$$


From this representation one can see at once that $f^{\prime} \in H^{p}$ for some $p>0$ (namely for all $p<1 / 2$, see e.g. [12], p. 80), so that $f^{\prime}\left(e^{i \theta}\right)$ exists for almost all $\theta \in[0,2 \pi]$ and

$$
\lim _{r \rightarrow 1} \int_{0}^{2 \pi}\left|f^{\prime}\left(r e^{i \theta}\right)\right|^{p} d \theta=\int_{0}^{2 \pi}\left|f^{\prime}\left(e^{i \theta}\right)\right|^{p} d \theta .
$$

For to get a sharp $H^{p}$-result for a polygonal mapping $f$ depending only on the parameters of the Schwarz-Christoffel formula, hence on the geometry of the image surface of $f$, we assume without loss of generality that $\mu_{k}>0(k=1, \ldots, m)$ and $\nu_{k}:=-\mu_{k+m}>0(k=1, \ldots, n-m)$ and write $y_{k}:=x_{k+m}(k=1, \ldots, n-m)$. Then

$$
f^{\prime}(z)=\prod_{k=1}^{n} \frac{1}{\left(1-\overline{x_{k}} z\right)^{2 \mu_{k}}}=\frac{\prod_{k=1}^{n-m}\left(1-\overline{y_{k}} z\right)^{2 \nu_{k}}}{\prod_{k=1}^{m}\left(1-\overline{x_{k}} z\right)^{2 \mu_{k}}},
$$

and so

$$
\left|f^{\prime}(z)\right| \leq \frac{\prod_{k=1}^{n-m} 2^{2 \nu_{k}}}{\prod_{k=1}^{m}\left|1-\overline{x_{k}} z\right|^{2 \mu_{k}}} .
$$

As $\sum_{k=1}^{n-n} \nu_{k}<\sum_{k=1}^{n}\left|\mu_{k}\right|=: K / 2$ we get

$$
\left|f^{\prime}(z)\right|<2^{K} \frac{1}{\prod_{k=1}^{m}\left|1-\overline{x_{k}} z\right|^{2 \mu_{k}}} .
$$

By (75) we have to check the finiteness of

$$
\int_{0}^{2 \pi} \frac{d \theta}{\prod_{k=1}^{m}\left|1-\overline{x_{k}} e^{i \theta}\right|^{2 \mu_{k} p}}
$$

Therefore suppose without loss of generality that $x_{k}(k=1, \ldots, m)$ are ordered successively on $\partial \mathrm{D}$ and define $\left(x_{m+1}:=x_{1}\right)$

$$
d:=\min \left\{\operatorname{dist}\left(x_{k}, x_{k+1}\right) \mid k=1, \ldots, m\right\} .
$$

Clearly $d>0$ as the points $x_{k}(k=1, \ldots, m)$ are isolated. (On the other hand the value of $d$ depends heavily on $n$ and for all sequences $\left(x_{k}\right)_{k \in N}$ of unimodular numbers $d \rightarrow 0$ as $n \rightarrow \infty$.) Now we decompose the integral (77) in $m$ components. Choose $t_{k}:=\frac{1}{2}\left(\arg \left(x_{k}\right)+\arg \left(x_{k-1}\right)\right)(k=1, \ldots, m)$, and observe that

$$
\left|1-\overline{x_{k}} e^{i \theta}\right|=\left|e^{i \theta}-x_{k}\right|>\frac{d}{2} \quad(k=1, \ldots, m)
$$

for $\theta \notin\left[t_{k-1}, t_{k}\right],\left(t_{m+1}:=t_{1}\right)$. Now it follows for $j=1, \ldots, m$ that

$$
\begin{aligned}
\int_{t_{j}}^{t_{j+1}} \frac{d \theta}{\prod_{k=1}^{m}\left|1-\overline{x_{k}} e^{i \theta}\right|^{2 \mu_{k} p}} & <\left(\frac{2}{d}\right)^{K p} \cdot \int_{t_{j}}^{t_{j+1}} \frac{d \theta}{\left|1-\overline{x_{j}} e^{i \theta}\right|^{2 \mu_{j} p}} \\
& \leq\left(\frac{2}{d}\right)^{K p} \cdot \int_{0}^{2 \pi} \frac{d \theta}{\left|1-\overline{x_{j}} e^{i \theta}\right|^{2 \mu_{j} p}},
\end{aligned}
$$


which is finite if and only if $p<1 /\left(2 \mu_{j}\right)$. So (77) is finite iff $p<1 /\left(2 \mu_{\max }^{+}\right)$, where $\mu_{\max }^{+}=\max \left\{\mu_{k} \mid k=1, \ldots, n\right\}$. This gives

LEMMA 11 Let $f$ be a Schwarz-Christoffel mapping. Then $f^{\prime} \in H^{p}$ for all $p<$ $1 /\left(2 \mu_{\max }^{+}\right)$, and this bound is sharp, i.e.

$$
\operatorname{dim}_{H F}\left(f^{\prime}\right)=\frac{1}{2 \mu_{\max }^{+}} .
$$

Analogously one gets for $1 / f^{\prime}$

LEMMA 12 Let $f$ be a Schwarz-Christoffel mapping. Then $1 / f^{\prime} \in H^{p}$ for all $p<$ $1 /\left(\alpha_{\max }-1\right)$, and this bound is sharp, i.e.

$$
\operatorname{dim}_{H^{p}}\left(1 / f^{\prime}\right)=\frac{1}{\alpha_{\max }-1} .
$$

Proof The same procedure as above shows that $1 / f^{\prime} \in H^{p}$ for all $p<1 /\left(2 l^{\prime}\right.$ max $)$ where $l^{\prime} \max :=\max \left\{v_{k} \mid k=1, \ldots, n \quad m\right\}$. By (9) it follows that $2 l^{\prime}{ }_{\max }=-\left(1-\alpha_{\max }\right)$.

\section{INTEGRAL MEANS FOR FUNCTIONS OF BOUNDED BOUNDARY ROTATION}

For functions of bounded rotation $K \pi$ we have the usual representation (34)

$$
\frac{f^{\prime \prime}}{f^{\prime}}(z)=-2 \int_{\partial \mathbf{D}} \frac{d \mu(x)}{z-x}
$$

for some signed measure $\mu$ with Lebesgue decomposition $\mu=\mu_{\mathrm{disc}}+\mu_{\text {cont }}$. Then $\mu_{\text {disc }}=\sum_{k=1}^{\infty} \mu_{k} \delta_{x_{k}}$ for $x_{k} \in \partial \mathrm{D}(k \in \mathrm{N})$ and $\sum_{k=1}^{\infty}\left|\mu_{k}\right| \leq K / 2$. Let now $\epsilon>0$ be given and choose $m \in N$ large enough that

$$
\sum_{k=m+1}^{\infty}\left|\mu_{k}\right| \leq \epsilon
$$

and that the maximal value $\mu_{\max }=\left|\mu_{k_{0}}\right|$ is attained for $k_{0} \leq m$. We write $y_{k}:=x_{k}$, $(k>m)$ and get

$$
\frac{f^{\prime \prime}}{f^{\prime}}(z)=-2 \sum_{k=1}^{m} \frac{\mu_{k}}{z-x_{k}}-2 \sum_{k=m+1}^{\infty} \frac{\mu_{k}}{z-y_{k}}-2 \int_{\partial \mathbf{D}} \frac{d \mu_{\text {cont }}(x)}{z-x} .
$$

For the last expression we write

$$
-2 \int_{\partial \mathbf{D}} \frac{d \mu_{\text {cont }}(x)}{z-x}=: \frac{k^{\prime \prime}}{k^{\prime}}(z)
$$

so that an integration gives (without loss of generality $f$ is always assumed to be normalized by (1)),

$$
f^{\prime}(z)=\prod_{k=1}^{m} \frac{1}{\left(1-\overline{x_{k}} z\right)^{2 \mu_{k}}} \cdot \prod_{k=m+1}^{\infty} \frac{1}{\left(1-\overline{y_{k}} z\right)^{2 \mu_{k}}} \cdot k^{\prime}(z)
$$


Now we go on as in the case of polygonal functions. Suppose without loss of generality that $x_{k}(k=1, \ldots, m)$ are ordered successively on $\partial D$ and define (now set $\left.x_{m+1}:=x_{1}\right) d>0$ by (78). Choose $t_{k}:=\frac{1}{2}\left(\arg \left(x_{k}\right)+\arg \left(x_{k-1}\right)\right)(k=1, \ldots, m)$ so that (79) holds for $\theta \notin\left[t_{k-1}, t_{k}\right],\left(t_{m+1}:=t_{1}\right)$.

Suppose now that $k^{\prime}=1$. Then it follows for $j=1, \ldots, m$ that

$$
\begin{aligned}
& \int_{t_{j}}^{t_{j+i}}\left|f^{\prime}\left(e^{i \theta}\right)\right|^{P} d \theta=\int_{t_{j}}^{t_{j+1}} \frac{d \theta}{\prod_{k=1}^{n t}\left|1-\overline{x_{k}} e^{i \theta}\right|^{2 \mu_{k} p} \cdot \prod_{k=m+1}^{\infty}\left|1-\overline{y_{k}} e^{i \theta}\right|^{2 \mu_{k} p}} \\
& <\left(\frac{2}{d}\right)^{K p} \cdot \int_{t_{j}}^{t_{j+1}} \frac{d \theta}{\left|1-\overline{x_{j}} e^{\left.i \theta\right|^{2 \mu_{j} p}} \cdot \prod_{k=m+1}^{\infty}\right| 1-\overline{y_{k}} e^{\left.i \theta\right|^{2 \mu_{k} p}}}
\end{aligned}
$$

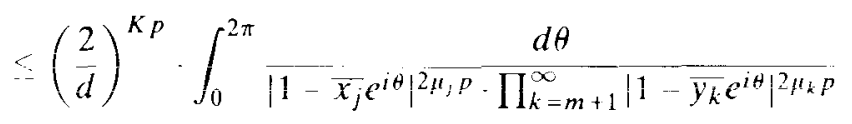

$$
\begin{aligned}
& \leq\left(\frac{2}{d}\right)^{K_{F}} \cdot \int_{0}^{2 \pi} \frac{d \theta}{\left|1-\overline{x_{j}} e^{i \theta}\right|^{2} P\left(n_{j}+t\right)}
\end{aligned}
$$

by (80) (for the last step see also [12], p. 80) which is finite if and only if $p<$ $1 /\left(2\left(\mu_{j}+\epsilon\right)\right)$. As $f$ was arbitrary we see that $f^{\prime} \in H^{P}$ for all $p<1 /\left(2 \mu_{\text {nax }}^{+}\right)$where $\mu_{\text {max }}^{+}:=\max _{k \in \mathbb{N}} \mu_{k}$ as in the polygonal case. This gives

THEOREM 11 Let $f \in V(K)$ with $f(\mathrm{D})=F$ such that $\partial F$ is linear except of a countable number of vertices $w_{k}=\int\left(x_{k}\right)$ of outer angle $2 \mu_{k} \pi(k \in \mathrm{N})$. Then

$$
\operatorname{dim}_{H^{p}}\left(f^{\prime}\right)=\frac{1}{2 \mu_{\max }^{+}}
$$

and

$$
\operatorname{dim}_{H^{F}}\left(1 / f^{\prime}\right)=\frac{1}{\alpha_{\max }-1}
$$

The result given here holds also if the function $k^{\prime}$ defined by (81) is bounded in $\mathrm{D}$. We conjecture that $(82)-(83)$ hold for all functions of bounded boundary rotation. Theorem 11 should be compared with results of Warschawski and Schober who showed the validity of (82) and (83) firstly for bounded univalent functions of bounded boundary rotation whose boundary curves $\partial f(D)$ are furthermore of bounded arc length-chord length ratio and secondly for bounded univalent functions whose ranges have only a finite number of vertices and for which some further technical conditions hold ([36], Theorems 2 and 3). We remark that our result does not at all depend on boundedness or univalence.

\section{INTEGRAL MEANS FOR CONVEX FUNCTIONS WITH VANISHING SECOND COEFFICIENT}

For convex functions the results of the last section apply. Moreover we get for functions with vanishing second coefficient 
THEOREM 12 Let $m \in N$ and $f_{m} \in K$ of form (66). Then $f_{m}^{\prime} \in H^{p}$ for all $p<m / 2$. This result is sharp for the convex function $f$ with $f^{\prime}(z)=1 /\left(1-z^{m}\right)^{2 / m}$.

Proof By $(68)$ in the given situation $f_{m}^{\prime}(z) \prec 1 /(1-z)^{2 / m}=: F^{\prime}(z)$, so that the result follows by the Littlewood subordination theorem as $1 /(1-z)^{\alpha} \in H^{p}$ for all $p<1 / \alpha$.

For $f^{\prime}(z)=1 /\left(1-z^{m}\right)^{2 / m}=F^{\prime}\left(z^{m}\right)$ we have

$$
\int_{0}^{2 \pi}\left|f^{\prime}\left(r e^{i \theta}\right)\right|^{p} d \theta=\int_{0}^{2 \pi}\left|F^{\prime}\left(r e^{i m \theta}\right)\right|^{p} d \theta=\int_{0}^{2 \pi}\left|F^{\prime}\left(r e^{i \theta}\right)\right|^{p} d \theta
$$

where the last equation follows by the substitution $\theta \rightarrow m \theta$ and from the periodicity of the exponential function, so that the result is sharp.

As a corollary we have a generalization of Theorem $8(\mathrm{c})$.

COROLLARY 9 LeI $f(z)=z+a_{2} z^{2}+a_{3} z^{3}+\cdots \in K$ with $a_{2}=a_{3}=0$. Then $f^{\prime} \mathrm{C}$ $H^{1}$ and $f(D)$ has a rectifiable boundary.

Proof The theorem shows that $f^{\prime} \in H^{1}$. As $f$ is bounded by Theorem 8(c) (or by (73)) and $f(\mathrm{D})$ therefore is a Jordan domain, we get the conclusion.

We remark that the theorem is a special case of our conjecture as functions of the given form satisfy $2 \mu_{\max }^{+} \leq 2 / m$ (see Theorem 8 (a)).

\section{References}

[1] L. V. Ahlfors, Quasiconformal reflections, Acta Math. 109 (1963), 291-301.

[2] J. Becker, Löwnersche Differentialgleichung und quasikonform fortsetzbare schlichte Funktionen, J. Reine Angew. Math. 255 (1972), 23-43.

[3] J. Becker, Some incqualities for univalent functions with quasiconformal extensions, In: General Inequalities 2, edited by E. F. Beckenbach, Birkhäuser, Basel, 1980, 411-415.

[4] M. Biernacki, Sur la représentation conforme des domaines linéairement accessibles, Prace Mat.-Fiz. 44 (1936), 293-314.

[5] P. L. Duren, Theory of $H^{P}$-spaces, Pure and Applied Mathematics 38, Academic Press, New YorkLondon, 1970.

[6] P. L. Duren, Univalent functions, Grundlehren der mathematischen Wissenschaften 259, SpringerVerlag, New York-Berlin-Heidelberg-Tokyo, 1983.

[7] P. L. Duren, H. S. Shapiro and A. L. Shields, Singular measures and domains not of Smirnov type, Duke Math. J. 33 (1966), 247-254.

[8] U. Gall, Über das Randverhalten von Bazilevið̌-Funktionen, Dissertation an der Technischen Universität Berlin, 1986.

[9] G. M. Goluzin, Some bounds on the coefficients of univalent functions (in Russian), Mat. Sb. 3, 45 (1938), 321-330.

[10] G. M. Golusin, Geometrische Funktionentheorie, Hochschulbücher für Mathematik Band 31, VEB Deutscher Verlag der Wissenschaften, Berlin, 1957.

[11] W. K. Hayman, On functions with positive real part, J. London Math. Soc. 36 (1961), 35-48.

[12] D. J. Hallenbeck and T. H. MacGregor, Linear problems and convexity techniques in geometric function theory, Monographs and Studies in Mathematics 22, Pitman, Boston-London-Melbourne, 1984.

[13] W. Hengartner, A. Pfluger and G. Schober, On support points in the class of functions with bounded boundary rotation, Ann. Acad. Sci. Fenn. Ser. A. I. Math. 6 (1981), 213-224.

[14] W. Kaplan, Close-to-convex schlicht functions, Mich. Math. J. 1 (1952), 169-185.

[15] W. Koepf, Extrempunkte und Stützpunktc in Familieñ nichtverschwindender schlichter Funktionen, Complex Variables 8 (1987), 153-171. 
[16] W. Koepf, On the Fekete-Szegö problem for close-to-convex functions, Proc. Amer. Math. Soc. 101 (1987), 89-95.

[17] W. Koepf, On the Fekete-S\%gë problem for close-to-convex functions II, Arch. Math. 49 (1987), $420-433$.

[18] W. Koepf, Convex functions and the Nehari univalence criterion. In: Complex Analysis, Proc. of the XIII. Rolf Nevanlinna-Colloquium, Joensuu, August 1987, edited by I. Laine, S. Rickman and T. Sorvali, Lecture Notes in Mathematics 1351, Springer-Verlag, Berlin-Heidelberg-New York, (1988), 214-218.

[19] W. Knepf, On close-to-convex functions and linearly accessible domains, Complex Variables 11 (1989), 269-279.

[20] M. A. Lawrentjew und B. W. Schabat, Methoden der komplexen Funktionentheorie, Mathematik für Naturwissenschaft und Technik Band 13, VEB Verlag Deutscher Wissenschaften, Berlin, 1967.

[21] O. Lehto, Domain constants associated with Schwarzian derivative, Comm. Math. Helv. 52 (1977), 603-610.

[22] O. Lehto and K. I. Virtanen, Quasikonforme Abbildungen, Grundlehren der mathematischen Wissenschaften 126, Springer-Verlag, Bcrlin-Hcidclbcrg-Ncw York, 1965.

[23] J. E. Littlewood, Lectures on the theory of functions, Oxford University Press, London, 1944.

[24] A. Mukherjca and K. Pothoven, Real and Functional Analysis, Mathematical concepts and methods in science and enginecring 6. Plenum Press, New York-London, 1978.

[25] Z. Nehari, The Schwarzian derivative and Schlicht functions, Bull. Amer. Math. Soc. 55 (1949), 545551.

[26] Z. Nchari, A property of convex conformal maps, J. Anal. Math. 30 (1976), 390-393.

[27] V. Paatero, Über die konforme Abbildung von Gebieten deren Ränder von beschränkter Drehung sind., Ann. Acad. Sci. Fenn. Ser. A. I. Math. 33:8 (1931), 1-78.

[28] Ch. Pommerenke, On starlike and convex functions, J. London Math. Soc. 37 (1962), 209-224.

[29] Ch. Pommerenke, Linear-invariante Familien analytischer Funktionen I., Math. Ann. 155 (1964), 108-154.

[30] Ch. Pommerenke, Linear-invariante Familien analytischer Funktionen II., Math. Ann. 156 (1964), 226-262.

[31] Ch. Pommerenke, On close-to-convex analytic functions, Trans. Amer. Math. Soc. 114 (1965), 176186.

[32] Ch. Pommerenke, Univalent functions, Studia Mathematica/Mathematische Lehrbücher 25, Vandenhoeck \& Ruprecht, Göttingen, 1975.

[33] G. Schobcr, Univalent functions-selected topics. Lecture Notes in Mathematics 478, Springer-Verlag, Berlin-Heidelberg-New York, 1975.

[34] T. Sheil-Small, On lincarly accessible univalent functions, J. London Math. Soc. 6 (1973), 385-398.

[35] E. Study, Vorlesungen über ausgewählte Gegenstände der Geometrie, 2. Heft: Konforme Abbildung einfach-zusammenhängender Bereiche, Teubner-Verlag, Leipzig-Berlin, 1913.

[36] S. E. Warschawski and G. Schober, On conformal mapping of certain classes of Jordan domains, Arch. Rat. Mech. Anal. 22 (1966), 201-209.

[37] K.-J. Wirths, Über holomorphe Funktionen, die einer Wachstumsbeschränkung unterliegen, Arch. Math. 30 (1978), 606-612. 